\title{
Le rôle de la nouvelle dans la refondation du roman chevaleresque : le cas de l'Inamoramento de Orlando de Matteo Maria Boiardo
}

\section{Patrizia De Capitani}

\section{(2) OpenEdition \\ Journals}

Édition électronique

URL : http://journals.openedition.org/cei/169

DOI : $10.4000 /$ cei. 169

ISSN : 2260-779X

\section{Éditeur}

UGA Éditions/Université Grenoble Alpes

\section{Édition imprimée}

Date de publication : 15 mars 2010

Pagination : 81-114

ISBN : 978-2-84310-164-9

ISSN : 1770-9571

Référence électronique

Patrizia De Capitani, « Le rôle de la nouvelle dans la refondation du roman chevaleresque : le cas de I'Inamoramento de Orlando de Matteo Maria Boiardo », Cahiers d'études italiennes [En ligne], 10 | 2010, mis en ligne le 15 septembre 2011, consulté le 28 mars 2021. URL : http://journals.openedition.org/ cei/169 ; DOI : https://doi.org/10.4000/cei.169 


\title{
LE RÔLE DE LA NOUVELLE DANS LA REFONDATION DU ROMAN CHEVALERESQUE : LE CAS DE L'INAMORAMENTO DE ORLANDO DE MATTEO MARIA BOIARDO
}

\author{
Patrizia De Capitani \\ Université Stendhal - Grenoble 3
}

La nouvelle réaliste en prose que Boccace a portée à la perfection connaît une phase d'effacement temporaire en Italie au tournant $\mathrm{du} \mathrm{Xv}^{\mathrm{e}}$ siècle malgré les notables exceptions du Novellino de Masuccio Salernitano (I476) et des Porretane de Sabbadino degli Arienti (I483) I. Elle est concurrencée par des récits à caractère non réaliste où dominent le merveilleux, le fabuleux (fiabesco) et le romanesque. Ces récits existent autant sous la forme autonome des cantari, narrations brèves en ottava rima, que sous celle de contes enchâssés à l'intérieur d'un roman chevaleresque, genre qui parvient à maturité à cette époque grâce aussi à l'invention de l'imprimerie qui permet d'en fixer le texte, alors que, sous forme manuscrite, un genre ouvert par nature comme le roman chevaleresque permettait toutes sortes d'ajouts et de modifications ${ }^{2}$. À Boiardo revient traditionnellement le mérite d'avoir introduit un certain nombre de nouvelles dans l'action principale de son roman chevaleresque, l'Inamoramento de Orlando. Il a été ensuite imité par Francesco Cieco da Ferrara et par l'Arioste. De ce

I. Sur la nouvelle au xv $\mathrm{xv}^{\mathrm{e}}$ siècle en Italie, voir L. Di Francia, Storia dei generi letterari italiani. Novellistica, 2 vol., Milan, Vallardi, I924-I925, t. I, p. 423-576; la thèse du déclin de la nouvelle en prose au tournant $\mathrm{du} \mathrm{Xv}$ e siècle est avancée par M. Beer, "Alcune osservazioni sulla novella nell'Orlando innamorato", dans R. Bruscagli, A. Quondam (dir.), Tipografie e romanzi in Val Padana fra Quattro e Cinquecento, Ferrare, F. C. Panini, 1992. Plus généralement, sur la nouvelle, voir La Novella italiana, Atti del convegno di Caprarola (19-24 septembre 1988), Rome, Salerno Editrice, I989, 2 vol. Sur le roman, on consultera, F. Moretti (dir.), Il Romanzo, Turin, Einaudi, 3 vol., 200I-2002; U. Dionne, La Voie aux chapitres. Poétique de la disposition romanesque, Paris, Éditions du Seuil, 2008.

2. Voir R. Ankli, "Altobello: un eroe nuovo nella stampa più antica», dans, J. Gómez-Montero et B. König (dir.), Letteratura cavalleresca tra Italia e Spagna (da "Orlando» al "Quijote»), Salamanque, SEMYR, 2004, p. I45. Pour une liste des principaux incunables chevaleresques parus en Italie on se reportera à M. Villoresi, $L a$ Letteratura cavalleresca. Dai cicli medioevali all'Ariosto, Rome, Carocci, 2000, p. I38-I40. 
fait, l'œuvre de Boiardo constitue un point d'observation stratégique pour l'étude des rapports entre le roman chevaleresque et la nouvelle, c'est-àdire entre deux formes de même nature, quoiqu'on puisse d'entrée de jeu rappeler qu'elles diffèrent en de nombreux points : longue la première, brève la seconde; complexe le roman qui multiplie les actions et les digressions qui en retardent l'épilogue, élémentaire la nouvelle, fondée sur une action unique. On sait par ailleurs que dans le roman l'épilogue désigne le moment où se dénouent tous les nœuds qui se sont créés au cours de l'action romanesque, alors que la nouvelle se termine par une conclusion souvent inattendue ${ }^{3}$.

Si la critique s'accorde à reconnaître à Boiardo cette priorité dans l'intégration de la nouvelle au roman chevaleresque, elle s'empresse en général de souligner toute une série de difficultés relatives à l'individualisation des nouvelles dans le corps du roman, ainsi qu'à leur fonction et à leur sens par rapport à l'action principale. Ces difficultés sont propres à ce type de situation, mais le cas des nouvelles de l'Inamoramento est plus problématique. S'il est facile de repérer les sept nouvelles du Mambriano du Cieco da Ferrara, dont Giuseppe Rua a fait un recueil ${ }^{4}$, et les treize ou quinze nouvelles du Furioso - leur nombre varie selon les critiques' ${ }^{-}$, les limites entre nouvelle et roman sont plus floues dans le cas de l'œuvre de Boiardo du fait que l'auteur lui-même n'ait été précis ni dans l'indication qu'il s'agit de nouvelles ni dans le sens qu'il attribuait au mot lui-même. De cette incertitude de statut en découle une autre concernant précisément la fonction et le sens des nouvelles par rapport au roman qui les contient. Si ces liens sont clairs dans le cas de l'Orlando furioso, au point d'admettre qu'il est impossible d'en extraire les nouvelles sans préjudice pour le message véhiculé par l'œuvre, certains pensent que cela serait tout à fait envisageable pour celles de l'Inamoramento, qui ne semblent pas être porteuses d'un message spécifique ${ }^{6}$.

Nous nous proposons de reconsidérer ces opinions critiques en vue d'analyser les relations que les nouvelles de l'Inamoramento entretiennent avec la narration principale autant du point de vue structurel que sémantique. Pour ce faire, nous nous interrogerons sur le statut des nouvelles à l'intérieur de l'Inamoramento en nous concentrant sur les liens existant

3. B. Eikenbaum, "Sur la théorie de la prose», dans T. Todorov (dir.), Théorie de la littérature, Paris, Éditions du Seuil, I965, p. 202-203.

4. G. Rua, Novelle del Mambriano, Turin, Loescher, I888.

5. Voir, dans ce même volume, la contribution de G. Sangirardi, "Les nouvelles du Roland furieux».

6. A. Franceschetti, "La novella nei poemi del Boiardo e dell'Ariosto", dans La Novella italiana, op. cit., p. 839 . 
entre les digressions et l'action principale du roman. Nous reprendrons par la suite la question des sources des nouvelles et de leur adaptation au genre chevaleresque. Nous nous pencherons enfin sur la signification des nouvelles dans leur contexte spécifique et dans celui plus général de l'œuvre.

\section{Statut des nouvelles dans l'Inamoramento de Orlando}

Revenons pour commencer sur ce qu'est une nouvelle à l'intérieur d'un roman chevaleresque. Pour qu'on puisse l'individualiser comme telle, il faut que soient respectés au moins deux critères : qu'il y ait quelqu'un qui raconte quelque chose à quelqu'un d'autre qui l'écoute et que ce récit ait suffisamment d'autonomie par rapport à l'action principale qui le contient, au lieu d'être une simple dilatation ou excroissance de l'intrigue majeure 7 .

Avant de considérer les nouvelles de Boiardo à la lumière des deux critères énoncés, rappelons que la critique s'accorde désormais pour dire que l'Inamoramento comporte sept nouvelles, cinq dans le premier livre et deux dans le deuxième. Le groupe de nouvelles du premier livre comprend les récits de Rocca crudele ou de Stella et Marchino (I VIII, 27-52), d'Iroldo, Tisbina et Prasildo (I XII, 5-89), d'Albarosa et Polindo (I XIII, 29-46), de Leodilla et Ordauro (I XXI, 48-69; XXII, Io-60) et enfin d'Origille (I XXIX, 5-37); le groupe du livre deux ceux de Narciso (II XVII, 50-63) et de Doristella (II XXVI, 20-6I). En ce qui concerne ce dernier, on admet qu'il se prolonge dans le chant suivant (XXVII, 3-35), mais à notre avis il ne s'agit pas vraiment d'une suite de la nouvelle puisque dans cette deuxième partie, le temps de la nouvelle et celui de l'intrigue majeure coïncident, mais nous reviendrons sur ce point. Nous sommes donc confronté à des récits de longueur variable et qui se succèdent sans régularité ni aucun ordre précis.

En considérant maintenant les sept nouvelles à l'aune des deux critères établis, nous constatons que le premier est respecté pour six d'entre elles sur sept, avec toutefois quelques nuances significatives. La nouvelle de Rocca crudele est racontée à Ranaldo par une vieille femme qui s'avère être la veuve de Marchino, déjà mentionné, celle de Tisbina est

7. Nous ne retenons donc pas ici le critère de Franceschetti selon lequel les nouvelles du roman chevaleresque commencent et se terminent par un groupe plus ou moins long de strophes de huit vers (les ottave) qui se lisent de manière consécutive et qui concernent une histoire allant du début à la fin ("La novella», art. cité, p. 20). 
racontée par Fiordelisa à Ranaldo, celle de Leodilla par celle-ci à Orlando et Brandimarte, celle d'Origille par Uldarno à Orlando, celle de Narcisse par la Reine Calidora à Orlando et Brandimarte, celle de Doristella par celle-ci toujours à Orlando et à Brandimarte. Seule la nouvelle d'Albarosa et Polindo n'est pas racontée par un narrateur, car elle est lue directement par Ranaldo dans un livre qu'il a trouvé près de la sépulture d'Albarosa elle-même. Le narrateur, on le voit, est presque toujours de sexe féminin tandis que celui qui écoute est un homme, et plus précisément l'un des héros du roman, comme si la nouvelle - même si ceci reste à vérifier comportait un message particulier à leur intention. Quant au statut du narrateur par rapport aux faits relatés, il s'agit dans cinq cas de quelqu'un qui y a pris directement part, les exceptions étant représentées par la nouvelle d'Albarosa, qui n'est pas racontée, ainsi que par celle de Tisbina, Iroldo et Prasildo, dont la narratrice est étrangère aux événements qu'elle présente. En outre, par respect du principe de surprise qui est à la base de l'esthétique du roman chevaleresque, la révélation de l'identité du narrateur est toujours repoussée à la fin de la nouvelle ${ }^{8}$. La situation de Calidora, la narratrice de la nouvelle de Narciso, est toutefois un peu particulière. D'un côté, il est dit explicitement que la source des faits rapportés est Calidora elle-même, qui les a également racontés à Orlando. Mais d'un autre côté, avant d'arriver aux oreilles de l'illustre public courtisan auquel s'adresse Boiardo, ces faits ont été en quelque sorte mis en forme par le narrateur du roman lui-même, comme il est précisé à la fin du conte : "Cette histoire (telle que je vous l'ai contée)/ Du beau Narcisse et de sa mort étrange/ Fut entièrement et fidèlement racontée par elle [Calidora] / Au comte Orlando près de la source ${ }^{9}$.» Il reste à se demander s'il existe une relation quelconque entre le sujet de la nouvelle - la relecture qu'elle propose du mythe de Narcisse - et cette manière de la raconter par l'interposition du narrateur du roman. Si le cas de Calidora reste unique, il existe de nombreuses occasions où la voix du narrateur du roman se mêle, pour ne pas dire se superpose, à celle du narrateur de chaque nouvelle. Ce mélange des voix, qui concerne les sept récits du roman de Boiardo, est particulièrement évident dans la nouvelle de Tisbina et il se vérifie à

8. La seule exception à cette règle étant la narratrice de la nouvelle de Rocca crudele qui se présente comme telle dès le début du récit : «Hora nel tempo che a viver te avanza», dit la narratrice à Ranaldo, «In questo tempo te voglio contare/ Qual cagion fece la usanza ordinare» (I VIII, 27, v. 4, 7-8). Toutes nos citations d'après le roman chevaleresque de Boiardo proviennent de : M. M. Boiardo, L'Inamoramento de Orlando, édition critique par A. Tisssoni Benvenuti et C. Montagnani, Milan-Naples, Ricciardi Editore, 1999, 2 vol.

9. II, XVII, 63, v. I-4 : «E questa historia (qual io v'ho contata) / Del bel Narciso e di sua morte strana, / Lei tuta la narrò come era stata, / Al Conte Orlando, prèso ala fontana.» (C'est nous qui soulignons.) 
chaque fois que sont énoncées certaines vérités générales sur l'amour et sa puissance, lesquelles sont carrément des devises de l'auteur ${ }^{\mathrm{IO}}$.

Les motivations qui président à l'introduction de ces vastes digressions narratives nous semblent, quant à elles, relever de deux grandes catégories, celle du delectare et celle du récit exemplaire. Le delectare intervient dans les nouvelles de Tisbina et de Doristella, où le conte doit soit faire oublier l'ennui d'un long voyage à cheval (nouvelle de Tisbina : I XII, 4), soit ranimer une ambiance devenue morose (Doristella : II XXVI, 20), soit encore distraire le malheureux Brandimarte qui vient à nouveau d'égarer sa bien-aimée Fiordelisa (nouvelle de Leodilla - première partie : I XXI, 49 ${ }^{\text {II. }}$. En ce qui concerne la deuxième partie de cette nouvelle, c'est Orlando lui-même qui, ayant fait monter Doristella sur son cheval, situation courante dans l'Inamoramento, prie celle-ci de reprendre le récit interrompu au chant précédent ${ }^{\mathrm{I} 2}$. Les nouvelles de Rocca crudele, d'Albarosa, d'Origille et de Narciso sont introduites pour expliquer une coutume cruelle ou des comportements étranges. Nous constatons donc que les sept digressions de l'Inamoramento respectent, à une exception près, le premier des deux critères que nous avons retenus pour les qualifier de nouvelles. Elles comportent trois autres particularités propres à la nouvelle : la brevitas - dans leur ensemble, elles ne vont pas au-delà d'un nombre limité de strophes et, à l'exception des nouvelles de Leodilla et Doristella, sont généralement contenues à l'intérieur d'un chant -, l'agrément et, pour certaines d'entre elles, l'exemplarité. Examinons maintenant leur conformité à l'autre principe que nous avons indiqué, c'est-à-dire leur degré d'autonomie par rapport à l'action du roman qui les contient. Pour cela il convient de considérer les liens qui se tissent entre la nouvelle et le roman au niveau des personnages, des coordonnées spatio-temporelles et du rapport s'établissant entre l'action majeure et l'action secondaire au moment de la conclusion de la nouvelle, lorsque le narrateur de cette dernière se tait pour redonner la parole à celui du roman.

En ce qui concerne les personnages, nous observons qu'à la seule exception de la nouvelle de Narciso, il y a passage de personnages de l'histoire

IO. «Perché descriver non si può lo amore/ A chi nol senti e a cui non l'ha nel core» (I XII, IO, v. 7-8); "Ché la vertù crescie sempremai / Che se ritrova l'homo inamorato" (I2, v. 3-4); "Perché sempre enterviene, in veritate, / Che la altereza è gionta con beltate» ( $\mathrm{I} 3, \mathrm{v} .7-8)$. D'autres exemples de superposition de la voix du narrateur du roman à celle du narrateur de la nouvelle dans I XXII (nouvelle de Leodilla) : I8, v. 7-8; 19, v. I-2; 29, v. 7-8; 32, v. 5-8; 42, v. 3-4; I XXIX (nouvelle d'Origille) : I3, v. 3-4.

II. "Sì che io verrò con voi, e con diletto / La mia novella voglio seguitare", dit Leodilla à ses interlocuteurs (I XXI, 69, v. 5-6). (C'est nous qui soulignons.)

I2. "In croppa la portava il conte Orlando, / E dolcimente la prese a pregare/ Che gli contasse, cossì caminando, / Quel che promisso avia di ragionare» (I XXII, IO, v. I-4). 
au roman et vice-versa. Certes cet échange se réalise de manière plus ou moins substantielle, ce qui confere à celui-ci des significations et des valeurs diverses. L'échange peut en effet se limiter à la simple mention d'un personnage que l'on retrouve par la suite dans l'action du roman. Tel est par exemple le cas du roi d'Orgagna appelé au secours par la femme de Marchino afin qu'il rétablisse la justice après la trahison de Marchino à l'encontre de Grifone. Ce personnage, mentionné pour des raisons d'ordre politique et géographique, n'établit aucun lien entre les deux sphères narratives. Il en va de même pour la nouvelle d'Iroldo et de Prasildo, malgré le transfert de ces deux héros qui après être intervenus dans la nouvelle se retrouvent dans la narration principale. Ce transfert n'est en effet qu'apparent, car, paradoxalement, il se fait au prix d'une coupure nette avec la nouvelle. Non seulement Tisbina, l'enjeu de la nouvelle et de la compétition courtoise entre les deux chevaliers, est exclue de ce transfert, mais l'Iroldo et le Prasildo qui intègrent le roman n'ont plus rien à voir avec les héros de la nouvelle. Il n'y a plus chez eux la moindre trace de la rivalité amoureuse qui les opposait dans la nouvelle; au contraire, ils sont désormais unis par une parfaite amitié. Iroldo est en effet surpris par Ranaldo en train de pleurer dans un bosquet parce qu'il a mis sans le vouloir Prasildo en danger de mort (Inamoramento, I XVII). La radicale transformation de leurs rapports et l'abolition de leur côté courtois et galant, symbolisé par l'évacuation de Tisbina, est un signe manifeste de rupture avec la nouvelle. Une fois intégrés dans le flot de la narration romanesque, Iroldo et Prasildo se fondent dans la foule de comparses chevaleresques qui peuplent le roman. Mais avant d'en arriver là, l'histoire émouvante de leur amitié a pris la place de la rivalité amoureuse de la nouvelle et fait l'objet d'une pause lyrique dans le flux ininterrompu de la narration. Ce type de pause, fréquente dans le roman de Boiardo, auteur par ailleurs des Amorum Libri Tres, le chansonnier lyrique le plus important du Quattrocento, renseigne opportunément le public sur le nouveau tournant qu'ont pris les rapports entre Iroldo et Prasildo en les séparant définitivement du contexte courtois de la nouvelle dont ils ont été les héros avec Tisbina.

Le lien qui s'établit entre les personnages de la forme brève et la forme longue du roman dans les quatre autres nouvelles de l'Inamoramento répond à des critères différents de ceux que nous venons de voir. Les nouvelles d'Albarosa et d'Origille comportent de fortes analogies entre elles, car toutes les deux présentent deux personnages hautement négatifs qui détournent les principaux idéaux chevaleresques et courtois. Ces deux personnages, respectivement Trufaldino, au nom évocateur, et Origille, le modèle même de la male pucele qui joue de mauvais tours à tous les che- 
valiers qu'elle croise, passent des deux nouvelles dont ils sont les héros à l'action du roman où ils s'illustrent par leurs méfaits. À la différence d'Iroldo et Prasildo qui, sortis du cadre de leur nouvelle, perdent tout relief et toute autonomie dans le roman, Trufaldino et Origille, une fois intégrée l'action du roman, y ont une place et une fonction significatives. Le premier est au cœur de l'épisode du siège d'Albraca qui s'étend sur plusieurs chants du premier livre et Origille revient à maintes reprises dans le livre II où elle ne cesse de se moquer d'Orlando qui a un fort penchant pour elle. Elle a également une part déterminante dans un épisode essentiel du livre II, celui où sont révélées les origines familiales et sociales de Brandimarte. Les nouvelles d'Albarosa et d'Origille sont donc étroitement dépendantes de l'action romanesque à laquelle elles fournissent deux personnages importants tant au niveau structurel que sémantique. Ajoutons que Trufaldino existe déjà dans la narration principale avant d'intervenir dans le récit qui ne fait que focaliser l'attention du public sur un personnage qui va par la suite jouer les premiers rôles ${ }^{13}$. Une fois rattaché à l'action principale, Trufaldino, tout comme Origille, y transfere son caractère de noirceur absolue, poursuivant dans le roman la série de traîtrises entamées dans la nouvelle.

L'insertion dans l'action du roman de Leodilla et Doristella, toutes les deux à la fois héroïnes et conteuses des faits qu'elles relatent, sert au dévoilement de l'origine familiale et sociale de Brandimarte et de Fiordelisa, personnages qui comptent parmi les nouveautés les plus marquantes introduites par Boiardo dans la narration chevaleresque. Ils ne relèvent en effet ni de la tradition carolingienne ni de celle arthurienne. Brandimarte est un guerrier païen qui a toutes les qualités du parfait chevalier chrétien, une sorte d'Orlando qui ne s'est pas laissé aveugler par l'amour. Fiordelisa aussi est une héroïne neuve dans la mesure où elle se situe au milieu entre la perfection d'une Bradamante - on se souviendra que la beauté de Ranaldo endormi lui fait pour un instant oublier Brandimarte - et la coquetterie perverse de certaines dames de la tradition arthurienne. Une fois advenu le dévoilement des origines de ces deux héros, Leodilla et Doristella sortent de scène sans plus jamais réapparaître, car leur débordement dans l'intrigue romanesque est uniquement fonctionnel au dévoilement de l'identité de Brandimarte et Fiordelisa. En revanche, à la différence des nouvelles d'Origille et de Trufaldino, il n'y a pas de lien

13. Trufaldino intervient pour la première fois dans le chant X du livre I; il combat du côté de Sacripante contre Agricane qui a assiégé le royaume d'Angelica. Trufaldino est d'entrée de jeu présenté comme un traître : «Re Trufaldino, il falso traditore» (I X, 40, v. 8). 
explicite entre les histoires racontées par Leodilla et Doristella, deux beffe dans la lignée du Décaméron aux dépens de deux maris vieux et jaloux, et le rôle que ces dernières jouent dans l'intrigue majeure. Cependant, en ce qui concerne Leodilla, le narrateur de l'Inamoramento souligne le ressentiment durable de celle-ci à l'encontre d'Orlando, qui non seulement passe une nuit entière à ses côtés sans essayer de la séduire mais qui, pire encore, n'arrête pas de ronfler en se montrant insensible à la présence de la dame (I XXIV, I5). La subtile allusion à la vertu légère de Leodilla est une manière de souligner la continuité existant entre le personnage de la nouvelle et celui du roman. Leodilla a beau avoir intégré la narration principale, elle garde intacte la propension à la jouissance physique qui la caractérisait dans la nouvelle.

Le temps de la nouvelle est-il détaché de celui de la narration principale? La nouvelle raconte un événement passé et abouti par rapport à l'action du roman où se trouve le personnage qui écoute le récit. Rappelons en effet que ce caractère d'aboutissement est un trait qui assure la distinction entre la nouvelle et le roman. Les intrigues des nouvelles de l'Inamoramento, commencées dans le passé, se dénouent aussi généralement dans le passé par rapport au récit principal. Les seules exceptions à cette règle nous semblent être la nouvelle de Rocca crudele et celle d'Albarosa et Polindo. Ainsi la première trouve-t-elle un épilogue au sein de la narration majeure dans la mesure où Ranaldo engage un combat contre le monstre né de l'union contre-nature de Marchino avec le cadavre de Stella, ce qui met en relation le présent de la narration principale avec le passé de la nouvelle. Ce lien entre passé et présent est effectué, dans la nouvelle d'Albarosa et Polindo, par le serment que Ranaldo fait sur le cadavre d'Albarosa de venger l'outrage qu'ont essuyé celle-ci et son amoureux Polindo. Serment que Ranaldo respecte en infligeant, plusieurs chants plus loin, un terrible supplice à Trufaldino, l'auteur du meurtre des deux amants (I XXVI, 46-49). Dans ce cas aussi, la conclusion de la nouvelle est pour ainsi dire provisoire et différée à un moment ultérieur qui se situe dans le roman. C'est seulement lorsque Ranaldo tue Trufaldino en l'attachant par les pieds à la queue de Rabicano qu'on peut estimer définitivement close l'histoire d'Albarosa et Polindo. Les deux nouvelles spéculaires de Doristella et Leodilla - autrement dit les beffe - ne sont pas non plus tout à fait terminées lorsque leurs deux héroïnes font leur entrée dans l'action du roman, car si elles ont réussi à se débarrasser de leurs vieux maris jaloux, elles doivent encore retrouver leurs amoureux, ce qui pour toutes les deux arrive plus tard dans l'action principale. La conclusion différée de ces deux récits s’explique par la nécessité de diluer les événements 
relatifs aux deux personnages jusqu'au moment où ils pourront jouer leur rôle dans le dévoilement du mystère des origines de Brandimarte et de Fiordelisa. La nouvelle d'Origille est elle aussi bel et bien terminée lorsque celle-ci fait son entrée dans l'action du roman. Mais la situation diffère de celle des nouvelles de Narciso et de Tisbina, Iroldo et Prasildo, les récits les plus autonomes à tous les égards par rapport à la narration principale, au point que la nouvelle de Tisbina, en particulier, comporte également une morale qui est énoncée explicitement par la narratrice. Au moins quatre des sept nouvelles de l'Inamoramento sont dépourvues d'une conclusion parce que leur dénouement est différé dans l'action du roman. L'option soit pour une conclusion aboutie, élément caractéristique de la nouvelle, soit pour une conclusion différée dans le temps est un indice du degré d'autonomie de la nouvelle par rapport au roman qui l'englobe.

Le tableau n'est pas plus homogène en ce qui concerne la manière dont s'opère le relais entre le narrateur du conte et celui du roman. Même si, dans la plupart des cas, le narrateur du roman reprend la parole en la coupant au conteur de la nouvelle, les motivations de l'interruption sont variables. Cette dernière est généralement déterminée par un événement imprévu ${ }^{\mathrm{I4}}$, et dans un seul cas par un manque évident d'intérêt de la part de l'interlocuteur ${ }^{15}$. Dans la nouvelle d'Origille, c'est en revanche Origille elle-même qui coupe la parole au conteur en démentant tout ce que celui-ci a dit sur son compte afin qu'Orlando, auquel s'adresse le récit, la délivre du terrible supplice auquel l'a condamnée sa méchanceté (I XXIX, 38-39). Dans trois cas seulement, le narrateur du roman n'interrompt pas celui du récit. Dans la nouvelle de Rocca crudele, il prend le relais du conteur par l'intermédiaire de Ranaldo qui demande à celui-ci la permission d'affronter le monstre ${ }^{16}$. La nouvelle de Narciso constitue, comme nous l'avons déjà dit, un cas à part car le narrateur du roman joue d'entrée de jeu les intermédiaires entre le public de l'œuvre et Calidora qui raconte la nouvelle. Le récit d'Albarosa, enfin, n’est pas raconté mais

I4. «Parlava la dongella tutta fiata, / Quando davanti a lor, nel bosco folto, / Odirno una alta voce, e smisurata" (nouvelle de Tisbina, I XII 90, v. I-3) ; "Ma nova cosa che ebbe ad aparire/ Sturbò il ragionar dela dongiella: / Ché un cervo al verde prato vedean gire» (nouvelle de Leodilla, deuxième partie, I XXII, 57, v. I-3) ; «Ragionava in tal modo Doristela/ Et altre cose asai volea seguire, / Ché non era compita sua novela» (Doristella, II XXVI, 53, v. I-3).

I5. "Ma vui aveti forse altro che fare,/ Perché io vedo entrambi nelo aspeto/ Esser sospesi e de intorno guardare" (Leodilla première partie, I XXI, v. 2-4). Il est question de la gêne d'un auditeur face à un conteur peu habile, ce qui n'est pas forcément le cas de Leodilla, dans Décaméron VI, 6 où Madonna Oretta prie un chevalier d'interrompre une histoire qu'il raconte trop mal.

I6. "[Ranaldo] Disse: - Deh, matre, non mi far contesa: / Concedimi, per Dio, che dentro vada/ Armato come io sono e con la spada" (I VIII, v. 6-8). 
directement lu par Ranaldo dans un livre et par ailleurs il est laissé en suspens car Albarosa est encore vivante lorsqu'il s'interrompt ou, plus vraisemblablement, lorsque Ranaldo en interrompt la lecture, accablé par l'émotion. Il existe donc bien une certaine variété dans les motivations qui règlent le passage de témoin entre le narrateur du récit et celui du conte. L'interruption narrative qui préside au transfert n'est pas toujours due à un fait surprenant, comme l'imposerait l'esthétique de la surprise qui domine, selon Bakhtine, dans la narration chevaleresque ${ }^{17}$. Cette technique, assurément, reste majoritaire, mais Boiardo n'hésite pas à recourir à des motivations qui proviennent de la nouvelle pour marquer ce passage de témoin narratif.

Les sept nouvelles de l'Inamoramento n'ont pas toutes le même statut par rapport au roman qui les contient. Quatre - Leodilla, Doristella, Albarosa et dans une moindre mesure Origille - sont si étroitement dépendantes de la narration majeure qu'elles pourraient bien avoir été introduites après coup. Les trois autres - Tisbina, Narciso et aussi Rocca crudele - semblent garder une autonomie à l'égard du roman qui les abrite.

Nous allons maintenant procéder à l'examen des sept nouvelles de l'Inamoramento que nous classerons, par commodité, en trois groupes: les nouvelles de trahison (Rocca crudele, Albarosa et Polindo, Origille), les nouvelles axées sur une beffa (nouvelles de Leodilla et de Doristella) et enfin celles de Tisbina et de Narciso qui n'appartiennent à aucune catégorie précise.

\section{Les nouvelles de trahison}

Dans Rocca crudele, la première des nouvelles de l'Inamoramento, est racontée la trahison perpétrée par Marchino à l'encontre de son voisin Grifone. Cette nouvelle, comme les deux autres de ce premier groupe, est introduite par une scène impressionnante qui se présente à Ranaldo. Avant de parvenir à Rocca crudele, Ranaldo traverse une "selva oscura", souvenir dantesque toujours annonciateur de mort ou d'une épreuve terrible dans le roman de Boiardo. Le spectacle d'horreur sur lequel il tombe à son issue de la selva et après avoir été fait prisonnier par un géant est décrit avec une grande complaisance : les parois de la forteresse où le géant le conduit sont rougies par le sang et tapissées de membres humains tout fraîchement 
séparés des corps auxquels ils étaient unis. La raison de cette horrible mise en scène est dévoilée seulement à la fin de la nouvelle, selon une technique qui revient dans les deux autres nouvelles de ce même groupe en vue de maintenir le suspens.

La trahison proprement dite est consommée dans la première partie de l'histoire : pris d'un soudain désir pour Stella, femme de Grifone, Marchino tue traitreusement ce seigneur qui s'était toujours montré hospitalier et courtois à son égard. Le cadre féodal où a lieu la trahison est soigneusement décrit : la forêt, qui est dans un premier temps un lieu de rencontre et de loisirs paisibles tels que la chasse, devient par la suite le lieu inquiétant où se déroule l'embuscade de Marchino. La Rocca d'Altaripa subit le même sort : lieu d'une sociabilité mondaine et courtoise - Grifone étant réputé pour son sens de l'hospitalité et sa franchise -, elle se transforme après le crime en un lieu de cauchemar comme l'indique sa nouvelle dénomination, Rocca crudele. En parlant de la soudaine passion de Marchino pour Stella, la narratrice qui est, rappelons-le, la femme de Marchino, évoque la "Disaventura», mais, en fait, l'accent narratif est aussitôt mis sur l'organisation impeccable du délit par Marchino qui n'a rien d'un amoureux égaré par la passion, mais qui incarne plutôt le criminel froid et déterminé. Avant de tendre l'embuscade fatale à Grifone, celui-ci pense même à en prendre les enseignes, ce qui facilitera sa pénétration dans la Rocca d'Altaripa, si bien gardée, après le meurtre de Grifone. L'usurpation des enseignes d'autrui était un très grave délit dans l'univers chevaleresque de l'Inamoramento, un crime évoqué à plusieurs reprises en divers endroits du roman et auquel est consacré toute la nouvelle d'Origille. Le moment clou de la trahison, lorsque Marchino entraîne Grifone dans le piège fatal, est dramatisé sous forme de dialogue entre la victime et son bourreau. Boiardo n'hésite pas à recourir au dialogue lorsqu'il veut introduire un élément de pathos ${ }^{18}$.

La deuxième partie du récit concerne la vengeance perpétrée contre Marchino tour à tour par la femme de celui-ci et par Stella, la femme de Grifone. C'est à ce moment-là qu'intervient la dimension horrifique, jusqu'alors absente. Pour représenter cette vengeance, Boiardo s'inspire d'Ovide, notamment de l'histoire de Procné et Philomèle (Métamorphoses, VI, 436-680). Il aggrave toutefois le caractère déjà atroce de l'histoire ovidienne par l'emprunt de détails horribles à d'autres sources antiques et

I8. Parmi les exemples les plus connus de dialogue il y a certainement celui du long échange entre Orlando et Agricane avant le duel meurtrier qui se solde par la mort de ce dernier, voir I XVIII, 40-48. 
de son époque ${ }^{19}$. Chez Boiardo, c'est la femme de Marchino elle-même qui raconte à la première personne et de manière détaillée comment elle a tué ses enfants qui imploraient sa pitié, en a décapité les cadavres et a fait cuire leurs chairs qu'elle a ensuite fait manger à Marchino. Admettre qu'une mère puisse trouver le courage de raconter de telles atrocités après les avoir commises est particulièrement insupportable. Ovide n'était pas allé aussi loin puisque le récit du meurtre de son enfant par Procné n'est pas raconté par elle mais par le narrateur. Toujours dans cette perspective outrancière, Marchino, pour se venger de Stella qui lui a révélé la vérité en lui montrant les têtes décapitées de ses enfants, s'unit avec elle en un accouplement monstrueux. Dans la description de ce dernier, les souvenirs de source ovidienne se mêlent à d'autres d'origine virgilienne, tout aussi atroces ${ }^{20}$. Comme s'il n'avait pas déployé assez d'horreurs, Boiardo fait porter des fruits à l'accouplement contre-nature de Marchino avec Stella : neuf mois après ces faits sanglants, un monstre cruel naît dans le caveau où se trouve le cadavre de Stella. C'est pour le nourrir, car il se nourrit de chair humaine comme son père, que Ranaldo et les autres victimes dont les membres tapissent les murs de Rocca crudele, ont été capturés.

Quel jugement porter sur cette nouvelle cruelle propre à engendrer un certain malaise? Boiardo est le premier à être conscient de ces excès puisque, aussitôt après avoir narré l'accouplement de Marchino avec le cadavre de Stella attaché à celui de Grifone, il introduit un petit commentaire sur les méfaits de Marchino qui marque sa prise de distance : "Je crois qu'il fit cela seulement pour se vanter qu'aucun homme ne saurait être plus scélérat que lui ${ }^{21}$.» Boiardo n'est pas un cas isolé à son époque. Des scènes d'une semblable atrocité se trouvent dans le Novellino de son contemporain Masuccio Salernitano, qui recourait à l'horreur dans une intention moralisatrice. En recourant à l'atrocité, Masuccio entendait stigmatiser les transgressions vis-à-vis de la morale et de la société dont se rendaient coupables certains personnages de ses nouvelles ${ }^{22}$. Mais Boiardo

19. Pour les sources antiques de la nouvelle de Rocca crudele, voir Inamoramento, éd. citée, note 25, p. 266. Plus généralement, sur les sources anciennes du roman de Boiardo, voir C. Zampese, "Or si fa rossa or pallida la luna ". La cultura classica nell'Orlando innamorato, Lucques, Pacini Fazzi, 1994.

20. Cet épisode est très probablement un souvenir de l'Énéide (VIII, 485-487) où il est question des abominations commises par le tyran Mézence qui «allait jusqu’à accoupler les vivants aux morts, les mains appliquées sur les mains, la bouche sur la bouche». Nous citons d'après Virgile, L'Énéide, traduite par M. Rat, Paris, Flammarion, 1965, p. I83.

2I. I VIII, 47, v. 7-8: "Credo io che il [Marchino] fece sol per darsi vanto/ Che altro hom non fosse scelerato tanto."

22. M. Papio, "Masuccio Salernitano's Gusto dell'orrido", dans G. Allaire (dir.), The Italian Novella. A Book of Essays, New York-Londres, Routledge, 2003, p. II9-I36. La nouvelle XXVIII du Novellino comporte justement ce gusto dell'orrido relevé par Papio comme nous pouvons le constater en lisant l' «Argument " introductif 
n'est pas un moraliste. La seule morale qui a pu être dégagée de cette nouvelle est interne à l'œuvre. L'aventure terrible de Rocca crudele est le prix que Ranaldo doit payer pour s'être enfui de Palazzo Gioioso, un lieu de délices et d'amour qu'Angelica lui avait apprêté. Cette sorte d'aventure terrible sert donc à punir la discourtoisie du baron. Par ailleurs, en mêlant les sources et en accumulant les excès, Boiardo finit par désamorcer l'horreur. Nous n'irons pas jusqu'à dire qu'il finit par susciter le sourire, mais il est difficile de croire qu'il s'attend à ce que cette nouvelle soit prise au sérieux.

La nouvelle d'Albarosa et Polindo, seconde de la série des nouvelles de trahison, est également introduite par une scène qui vise à impressionner le public. Ranaldo, encore lui, après avoir abattu un géant, parvient à une grotte dans laquelle est enchaîné Rabicano, cheval plus rapide que le vent. Rabicano n'est pas, toutefois, la seule merveille du lieu. Dans la grotte se trouve aussi une porte de marbre décorée de pierres précieuses qui encadre un catafalque sur lequel est déposée une dame morte. Nous sommes en présence d'un de ces objets riches et précieux qui sont récurrents dans l'Inamoramento, dont le rôle n'est pas seulement décoratif mais qui renvoient également au motif des richesses, lequel est doté d'une forte charge symbolique dans le roman chevaleresque. L'histoire de la dame se lit dans un livre écrit en caractères de sang qui se trouve à côté de la gisante. Tout comme dans Rocca crudele, le sang annonce ici une histoire tragique et terrible.

Si déjà le crime de Marchino dans la nouvelle précédente avait un mobile ténu, celui de Trufaldino n'en a aucun. À l'origine des délits présentés dans cette nouvelle, on trouve en effet la générique jalousie que Trufaldino, roi de Badaco (Bagdad), éprouve vis-à-vis d'un de ses vassaux, Orisello, qui est paré de toutes les qualités. Ici, à la différence de Rocca crudele, le traître attaque indirectement la victime. Pour frapper Orisello, Trufaldino profite en effet d'une faiblesse d'Albarosa, la sœur d'Orisello, c'est-à-dire de l'amour de celle-ci pour Polindo. Mais Albarosa, que Trufaldino a enfermée dans une forteresse avec Polindo pour faire pression sur Orisello, ne cède ni aux menaces ni aux tortures et préfere mourir plutôt que livrer son frère à Trufaldino. Si à la base de cette nouvelle il y a une histoire de traitrise, voire de félonie, elle est toutefois différente de la précédente à plusieurs égards. Tout d'abord, l'horreur y est très

de la nouvelle : «Un cavaliero provenzale ama soverchiamente la moglie, quale, da libidine assalita, si fa da uno nano carnalmente cognoscere; una mora de casa con una lancia tutti dui sul fatto le uccide; il marito le fa bottare per pasto de fere.» (Masuccio Salernitano, Il Novellino, G. Petrocchi éd., Florence, Sansoni, 1957, p. 264.) 
limitée et cantonnée à la scène, il est vrai plutôt choquante, où Trufaldino torture Albarosa qui refuse de lui livrer Orisello (I XIII, 43). Une seule scène ne paraît pas grand-chose, comparée à la suite d'horribles délits de la nouvelle de Rocca crudele. Les personnages de cette deuxième nouvelle ont également une certaine noblesse, notamment Albarosa, que n'avaient pas ceux de la précédente. On n'a plus trace ici du fort courant misogyne qui caractérisait la nouvelle de Rocca crudele; au contraire, le narrateur, c'està-dire Boiardo - rappelons-nous que cette nouvelle n'est pas racontée, mais directement lue par Ranaldo -, salue le courage de la dame à qui il a même attribué le nom évocateur d'Albarosa, association de pureté et de fragilité. Le narrateur laisse donc transparaître sinon de la sympathie au moins de la compassion à l'égard des deux victimes innocentes dont le seul tort a été de s'aimer à tel point qu'elles sont devenues imprudentes et naïves. Les allusions à l'imprudence et à la crédulité des deux amants ne visent ni les amoureux, ni leur passion, mais expriment le regret sincère du narrateur vis-à-vis du gâchis de la beauté et de la jeunesse ${ }^{23}$. Voir dans ces considérations une invitation à la prudence en amour serait peut-être aller trop loin, mais le ton indulgent et compatissant de certaines interventions de l'auteur nous autorise à y songer.

Enfin, si l'épreuve de Rocca crudele, propédeutique au terrible combat de Ranaldo avec le monstre issu de l'union entre Stella et Marchino, peut être interprétée comme la juste punition pour la discourtoisie du baron envers Angelica, celle d'Albarosa et Polindo, cinq chants après la précédente, marque la transformation de Ranaldo en héros épique. Alors qu'Orlando, tout pris par l'amour d'Angelica, a perdu de vue sa mission chevaleresque et guerrière, Ranaldo, rendu insensible à l'amour grâce à la source de Merlin, donne une suite au serment juré sur la tombe d'Albarosa et punit la félonie de Trufaldino. Ce châtiment clôt le long épisode du siège d'Albracà qui occupe la deuxième partie du livre I de l'Inamoramento. Pour secourir Angelica, Orlando se situe du côté de Trufaldino lors de ce siège; à Ranaldo reviennent donc les lourdes tâches de venger Albarosa en exécutant Trufaldino et de rétablir la justice plusieurs fois bafouée pendant l'épisode du siècle d'Albracà.

Orlando est justement l'interlocuteur d'Uldarno qui lui raconte la troisième nouvelle du groupe des récits de trahison et de félonie. Ainsi que les deux que nous avons déjà examinées, cette nouvelle est introduite par une mise en scène frappante et mystérieuse. Orlando, à peine sorti d'une selva

23. «Diversa cosa è lo amor veramente: / Teme ciascuno e crede ad ogni gente!» (I XIII, 36, v. 7-8); «Che altri mai che Polindo avrìa creduto / A quel malvagio mancator de fede?» (37, v. I-2). 
non qualifiée d'obscure, ce qui annonce une aventure dont le dénouement ne sera pas tragique, voit apparaître, au-delà d'un pont gardé par un chevalier, une dame suspendue à un arbre par les cheveux ${ }^{24}$. Orlando, qui se précipite au secours de la demoiselle, est arrêté dans son élan par le chevalier, Uldarno, qui lui raconte pourquoi Origille, la jeune fille suspendue, en est arrivée là. Origille, par pure méchanceté, s'est moquée de ses trois soupirants - Uldarno lui-même, Locrino et Ariante - et, ce qui est bien plus grave, a compromis leur honneur et a mis en danger leurs vies. Cette nouvelle, difficile à résumer tant les intrigues imaginées par Origille aux dépens de ses trois amoureux sont compliquées et gratuites, appartient au genre du récit misogyne dénonçant la volubilité et la légèreté féminines. Le supplice qui est infligé à Origille - osciller au vent comme une feuille, symbole de la légèreté et de l'instabilité - est la juste punition pour ces deux vices qu'elle possède au plus haut degré. Mais s'agit-il vraiment d'une nouvelle misogyne? La persévérance d'Origille à éconduire ses amants est tellement gratuite qu'on finit par s'amuser face aux machinations abracadabrantes ourdies par la jeune fille. Face à elle, les trois amoureux, si enclins à tomber dans le panneau, font piètre figure. Si le thème de cette nouvelle est la trahison, son esprit correspond plutôt à celui de la tromperie à l'encontre d'un naïf où le lecteur est forcé d'admirer l'astuce dont fait preuve le sexe faible. Cette impression est également confirmée par la conclusion de la nouvelle : si Origille subit le supplice de la suspension dans les airs, les amoureux qui ont enfreint le code chevaleresque par amour sont condamnés à la peine capitale. La menace n'est toutefois pas mise à exécution, car la nouvelle s'arrête avec la libération d'Origille par Orlando et rien n'est dit sur le destin des trois amoureux trop crédules. On peut dire alors que, sous couvert de misogynie, Boiardo, tout comme Boccace, célèbre l'astuce féminine. Nous pouvons en avoir confirmation en comparant la nouvelle d'Origille avec une de celles du Novellino de Masuccio qui met également en scène une jeune fille coquette et légère qui, comme Origille, éconduit plusieurs soupirants. La coquetterie de la jeune fille est lourdement sanctionnée par Masuccio, puisque celle-ci meurt de chagrin à la suite de la découverte de sa duplicitées. À l'inverse le double jeu d'Origille non seulement n'est pas sanctionné, mais c'est

24. "Alla rippa del fiume, ad un bel pino, / Stava una dama per le chiome impesa» (I XXVIII, 52, v. 3-4).

25. "Una iovenetta è amata da multi, e, uccellandoli, tutti li tiene in pastura; uno più che l'altri segue la pista; uno sclavo de la iovene la cognosce carnalmente, e al fervente amante il fa vedere; la iovene per dolore ne more, e l'amante compera lo sclavo e ponelo in libertà." (Masuccio Salernitano, Il Novellino, op. cit., nouvelle XXV.) 
grâce à lui qu'elle parvient à se faire détacher de l'arbre par Orlando ${ }^{26}$. Les discours plaintifs qu'elle lui tient pour le convaincre de son innocence et le décider à la décrocher de l'arbre ne sont cependant que mensonges. Une fois libérée, Origille s'arrange pour qu'Orlando devienne rapidement sa quatrième victime masculine; elle l'abandonne en prenant la fuite avec son cheval et en le laissant à pied ${ }^{27}$. Boiardo reprend la typologie de la nouvelle misogyne, mais en exagérant jusqu'aux limites de l'absurde les défauts du sexe féminin, il souligne l'absurdité des poncifs antiféministes. Est-il alors excessif de voir dans la nouvelle d'Origille un témoignage, certes indirect et à mots couverts, d'une réelle complicité du comte de Scandiano avec le sexe féminin parmi lequel il comptait de nombreuses admiratrices? Nous avons pu constater que même à l'intérieur d'une catégorie précise, celle des nouvelles de trahison, Boiardo fait preuve d'une certaine variété en jouant tantôt sur le registre de l'horreur et du pathos, tantôt sur celui de la misogynie qu'il parvient du reste à retourner à l'avantage du sexe féminin.

\section{La $b$ effa}

Les nouvelles racontées respectivement par Leodilla et par Doristella, qui en sont également les héroïnes, concernent deux beffe (tromperies) aux dépens de leurs maris vieux et jaloux. Ces deux nouvelles sont analogues quant au sujet et à la fonction par rapport à la narration principale. Comme nous l'avons en effet déjà expliqué, grâce à Leodilla et à Doristella les origines familiales et sociales de Brandimarte et Fiordelisa sont dévoilées.

Leodilla et Doristella, unies contre leur gré à deux vieillards jaloux, parviennent à se libérer de leur joug grâce à leur astuce et surtout à la collaboration de leurs amoureux. Ces deux histoires relatent les mauvais tours joués par deux femmes mal mariées à leurs époux jaloux dans l'esprit de la VII journée du Décaméron. Dans la nouvelle de Leodilla, la beffa aux dépens du vieux Folderico est précédée d'un préambule. Leodilla, fille du richissime roi Manodante, au nom suggestif, défie à la course - activité où elle excelle - ses deux soupirants, le jeune et beau Ordauro et le vieux et

26. "Cossì dicea la dama e con lamento / [...] / Per Dio pregando e sempre per pietate/ Che non la lassi in tanta crudeltate. // Non stete Orlando già molto a pensare, / Perché pietà lo mosse incontinente» (I XXIX, 39, v. $5-8 ; 40$, v. I-2).

27. "Il Conte non vi fece altro pensiero / [...] / Et ala dama lascia il suo destriero./ Lei, come gionto sopra il sasso il vede, / Forte ridendo disse: "Cavaliero, / Non sciò se seti usati a gir a piede, / Ma sciò ben dir che usar ve gli convene:/ Io vado in qua: Dio ve conduca bene!” (I XXIX, 5I, v. I-8.) 
rusé Folderico. Celui des deux qui réussira à la battre aura sa main, tandis que le perdant devra mourir. La jeune fille s'organise pour faire gagner Ordauro et faire perdre Folderico. Mais tout va à l'encontre des desseins de Leodilla, car le rusé Folderico, en laissant tomber d'un sac trois pommes d'or que celle-ci s'arrête à ramasser, gagne la compétition et obtient la main de la jeune fille. Celle-ci, pour sa part, lui promet une vie conjugale tourmentée par l'infidélité, et cette promesse introduit la deuxième partie de la nouvelle où il est proprement question de la beffa ourdie par Leodilla et Ordauro aux dépens de Folderico. Le préambule, comme nous avons pu le constater, constitue une relecture du mythe d'Atalante (Ovide, Métamorphoses, 560-696) à la lumière de l'interprétation allégorique de Boccace qui, dans sa Généalogie des dieux païens, considérait cette légende comme une illustration de la cupidité féminine ${ }^{28}$. Effectivement, le motif de la cupidité et du rapport aux richesses, qui joue un rôle capital dans l'Inamoramento, est également au cœur de la nouvelle de Leodilla. Il est intéressant de souligner qu'il est rendu actuel par des considérations d'ordre psychologique, social et moral susceptibles d'intéresser le public de Boiardo, notamment sa composante féminine.

Si l'Atalante ovidienne défie tous ses soupirants à la course pour échapper à des noces que les dieux lui annoncent funestes ${ }^{29}$, Leodilla veut d'un côté éviter le mariage avec quelqu'un qu'elle déteste ou qui lui serait imposé par la volonté paternelle ${ }^{30}$, de l'autre ne pas dévoiler ses sentiments à Ordauro, car ce serait contraire à la pudeur et socialement répréhensible ${ }^{3 \mathrm{I}}$. Par l'intermédiaire de Leodilla, le comte de Scandiano relève donc le double défi qui se présente aux jeunes filles amoureuses: d'un côté la soumission à la volonté paternelle et de l'autre la pudeur, entrave à laquelle Leodilla pense échapper par l'expédient de la course, source de tous ses malheurs. Cette pudeur excessive, ennemie du désir, n'est pas pour plaire à Boiardo qui célèbre dans l'amour l'union harmonieuse du corps et de l'âme. Il a donné sa plus belle célébration de cette conviction,

28. Inamoramento, op. cit., note 49, p. 6oI. Sur la nouvelle de Leodilla, voir F. Battera, «Per una lettura di Orlando Innamorato (I XX-XXII)", Studi e problemi di critica testuale, 34 (1987), p. 85-103; C. Searles, "The Leodilla Episode in Boiardo's Orlando Innamorato (I XX-XXII)», Modern Language Notes, XVII (I9O2), p. 330-342.

29. "Un jour qu'elle demandait à un oracle si elle devait prendre un époux : "Un époux! lui répond le dieu; non, Atalante, il ne t'en faut point; fuis le commerce d'un époux; et pourtant tu n'y échapperas pas et, sans cesser de vivre, tu cesseras d'être toi-même." "(Ovide, Métamorphoses, J.-P. Néraudau éd., Paris, Gallimard, I992, p. 34I.) L'oracle fait allusion à la métamorphose d'Atalante et de son époux en lions suite à l'ingratitude de celui-ci à l'égard de Vénus.

30. "Non era tuta mia la libertate,/ Però che il patre mio vi tenea parte» (I XXI, 53, v. I-2).

3I. "Vergogna rafrenò la voluntate/ Che presto in nave avrìa trato le sarte» (I XXI, 53, v. 3-4). 
nous le savons, dans la scène des ébats de Brandimarte et Fiordelisa, mais à chaque fois qu'il s'agit de célébrer la rencontre de deux amants, il est animé d'un souffle spécial. Ce souffle caractérise par exemple la description des retrouvailles de Doristella et Teodoro après une longue séparation ${ }^{32}$. Autre détail qu'il nous paraît intéressant de souligner, la remise en cause de la proverbiale astuce féminine. En effet, la machination de Leodilla échoue : la naïveté juvénile est mise à mal par la ruse du vieillard expérimenté, d'où le doute, exprimé par l'héroïne elle-même, sur la réalité de cette astuce attestée depuis Salomon ${ }^{33}$. Souvenons-nous qu'Atalante ramassait les pommes d'or qu'Hippomène lui lançait sous l'emprise d'une force supérieure, celle de la déesse Vénus. Leodilla, en revanche, est la victime de la ruse de Folderico, ainsi que de sa propre maladresse. La cupidité de Leodilla n'a en outre rien de prémédité. Elle est surprise en quelque sorte par le riche piège que lui tend le vieillard et ne sait pas y résister. La signification cachée sous l'image de la jeune fille qui ramasse la pomme d'or n'est pas tant l'avidité féminine que le contraste entre la préméditation du vieillard et la naïveté de la jeune fille. La machination juvénile de celle-ci échoue sous les coups de la ruse du vieillard expérimenté. Aussi le sens que prend sous la plume de Boiardo la relecture du mythe ovidien va-t-il bien au-delà de l'interprétation allégorique de Boccace, puisqu'elle s'enrichit d'observations d'ordre social et psychologique susceptibles d'intéresser ses contemporains, notamment son public féminin. Par sa relecture originale du mythe d'Atalante, Boiardo semble stigmatiser toute sorte de calcul qui s'opposerait à la spontanéité du désir et de la jeunesse, d'où le choix du motif de la beffa aux dépens d'un vieillard jaloux, le bouc émissaire préféré des jeunes premiers de la commedia erudita que l'Arioste s'apprêtait à faire revivre à Ferrare. Le théâtre, notamment le théâtre de Plaute, est très présent dans l'Inamoramento et en particulier, comme nous allons le voir bientôt, dans la nouvelle de Leodilla, ainsi que dans la narration principale ${ }^{34}$.

La nouvelle racontée par Doristella est le miroir de celle de Leodilla, même si elle n'a pas la même portée allégorique, notamment dans sa

32. II XXVII, I5, v. 6-7; I6 : «Lui conobe Doristela al primo guardo, / E lei conobe anco esso al primo trato,/ $[\ldots] /$ Però che ciascadun tanto s'amava/ Ch'altra sembranza non avea nel core./ [...]/ E ciascadun più streto s'abrazava/ Dandosi basi sì caldi d'amore/ Che ciascadun ch'intorno era in quel loco/ Morìan d'invidia, si parìa bel gioco!»

33. "Neli antichi proverbi dir se sòle/ Che malicia non è che donna avancie: / Salamon disse già queste parole, / Ma al nostro tempo se ritrovan ciance» (I XXI, 52, v. I-4). (C'est nous qui soulignons.)

34. La comédie des Captivi de Plaute est à l'origine de l'épisode de l'échange d'identité entre Orlando et Brandimarte qui a lieu dans le chant XII du livre II de l'Inamoramento. 
première partie. Doristella aime Teodoro qui l'aime aussi. Teodoro a été promis dès la plus tendre enfance à la sœur de Doristella (en l'occurrence Fiordelisa, comme nous le découvrirons plus tard), mais comme celle-ci a été enlevée - autre situation propre à la comédie antique - Teodoro est tombé amoureux de Doristella. Si Leodilla est en partie responsable de son mauvais sort, Doristella est la victime tout à la fois d'une certaine malchance et de la volonté paternelle. Alors que Teodoro s'apprête à demander la main de Doristella au père de celle-ci, le vieux et puissant Osbego lui emboîte le pas et obtient la jeune fille. La première partie dans chacune des deux nouvelles se termine donc sur la victoire provisoire des deux vieillards, dont les malheurs ne font toutefois que commencer car, ainsi que le laisse entendre Boiardo, il est absolument illusoire de croire qu'on peut s'opposer aux droits du désir, de l'amour et de la jeunesse.

Les nouvelles consacrent chacune leur deuxième partie à la représentation de la jalousie des deux vieillards. Tous les deux installent en effet leurs jeunes épouses dans des endroits inaccessibles et isolés. Osbego, soupçonnant que Doristella le trahit avec Teodoro, enferme la jeune fille dans un palais enchanté (II XXVII, 52). Folderico relègue Leodilla dans une tour au bord de la mer. Le motif de la femme recluse dans un lieu isolé est très répandu dans la nouvelle occidentale et Boiardo y trouve de quoi illustrer autant la folle jalousie du vieillard que son idéal de l'amour synthétisé dans la formule virgilienne "Omnia vincit amor ${ }^{35}$ ». Car Leodilla comme Doristella, la première aidée par Ordauro, la deuxième par un heureux hasard, parviennent à sortir de leurs prisons comme nous le verrons.

Dans la phase de la réclusion sont décrites de manière très savoureuse les craintes des deux jaloux et leurs précautions inutiles pour éviter que ne se réalise la promesse de cocuage faite autant par Leodilla que par Doristella lors du mariage. La représentation de la jalousie des deux vieillards frôle l'absurde dans les deux cas. Folderico est jaloux même des puces et des mouches - de sexe masculin - qui effleurent sa jeune épouse (I XXII, I7). Osbego, quant à lui, a beau être très prudent, il finit par confier la surveillance de Doristella à un serviteur borgne ("guerzo»), détail saugrenu et ironique. Comme nous pouvons le constater, le rire est la juste punition de la jalousie. Un autre motif commun aux deux nouvelles, et typique

35. Sur la réutilisation par Boiardo du motif de la femme recluse dans une tour, voir l'édition par G. Sinicropi de G. Sercambi, Novelle, Florence, Casa Editrice Le Lettere, I995, 2 vol., t. II, p. I2I8-I219. Sur la fortune de l' "Omnia vincit amor" dans la littérature amoureuse du Moyen Âge, voir la préface de E. Baumgartner à son édition Pyrame et Thisbé Narcisse Philomena, Paris, Gallimard, collection «folio classique», 2000, p. 8. 
de ce genre de situation, est l'impuissance des deux vieillards à satisfaire sexuellement leurs épouses qui s'en plaignent ouvertement ${ }^{36}$.

Malgré leurs précautions, les jaloux se laissent berner par les amoureux et nous entrons ainsi dans la troisième partie des deux novelles, celle où a lieu la beffa proprement dite. Dans les deux cas, la source du malheur est justement ce qui devait l'éviter. Dans la nouvelle de Folderico, la tromperie et l'infidélité conjugales se réalisent grâce à la tour où est enfermée la jeune femme, tandis que dans l'autre nouvelle le malheur vient du serviteur borgne Gambone qui faillit à sa tâche de surveillant de Doristella que lui a confiée Osbego. Dans les deux nouvelles la tromperie est organisée de manière à démontrer que les deux vieillards sont davantage les victimes de leur sottise que de l'astuce des amoureux.

Ordauro fait construire un palais à côté de la tour et parvient à jouir de Doristella grâce à un tunnel caché qui relie le palais à la tour et dont le vieux Folderico ne soupçonne pas l'existence. La situation est moins machiavélique dans l'autre nouvelle, puisque les deux amants profitent l'un de l'autre en l'absence d'Osbego. Il est explicitement dit que les amants concrétisent leurs envies grâce à l'argent. Ordauro dépense beaucoup pour faire construire son palais, et Teodoro utilise de l'argent pour corrompre Gambone qui le laisse ainsi jouir de Doristella ${ }^{37}$. Le motif de l'argent, déjà évoqué, surgit donc à nouveau dans ces deux nouvelles, démontrant l'importance que Boiardo lui accordait. Sous certaines conditions, amour et argent peuvent tout à fait faire bon ménage selon Boiardo. L'argent n'a pas le pouvoir d'acheter l'amour, contrairement à ce que pensent les deux vieillards des nouvelles, comme sans doute aussi d'autres vieillards bien plus réels. L'argent peut en revanche agrémenter, voire aider l'amour, mais seulement le vrai, celui qui naît d'une attirance naturelle et spontanée. En aucun cas il ne peut servir à susciter l'amour, car, comme le montrent ces deux nouvelles, les richesses sont sans pouvoir sur la nature et le désir. Mais s'il est vrai que la fortune aide les audacieux, elle aide encore mieux les

36. I XXII, I6, v. I-4 : «De zoglie e de oro e de ogni altro dileto / Era io fornita tropo a dismisura, / Fuor de il piacer che si prende nel leto, / De il qual avia più brama e magior cura. " Voir également à ce sujet la note 25 , p. 620 de notre édition de référence, où il est dit que la situation d'abstinence sexuelle de Leodilla comporte des analogies avec celle où Tristan tient son épouse Yseut aux Blanches Mains, mais Tristan le fait pour rester fidèle à son amour, alors que chez Boiardo le contexte est résolument grivois.

37. Leodilla dit explicitement que sans argent, Ordauro ne serait jamais parvenu à la libérer de l'emprise de Folderico : «Egli era rico di molto thesoro/ (Che sanza quel non val senno un lupino), / Onde con molto argento e con molto oro/ Fiè comprar un palagio in quel confino" (I XXII, I9, v. I-4); et Doristella : "Venne d'Harmenia in Bursa Theodoro/ [...] / Et ala via più presta s'attaccava, / Ché portato avìa seco assai tesoro; / Onde Gambon in tal modo aquetava" (II XXVI, 35, v. I-5). 
audacieux qui ont quelques moyens. Idéalisme et pragmatisme se côtoient dans la vision de l'amour du comte de Scandiano.

Il faut également souligner l'effort esthétique accompli par Boiardo pour rehausser l'intérêt de la beffa en renouvelant ainsi ce motif traditionnel de l'art narratif en prose. Dans la nouvelle de Leodilla, Boiardo y parvient en contaminant la nouvelle avec le Miles gloriosus de Plaute. Pour éconduire Folderico, Ordauro lui fait croire qu'il s'est marié avec la jumelle de Leodilla, alors que c'est Leodilla elle-même qui joue un double rôle, grâce à l'expédient du tunnel qui lui permet de changer rapidement de lieu, de disparaitre et de réapparaitre sous une autre identité. Cet expédient de la fausse jumelle est imaginé par l'esclave Palestrion dans le Miles de Plaute pour tromper le soldat fanfaron et permettre à Pleusiclès de jouir en paix de Philocomasie qu'il aime ${ }^{38}$. Dédoublements, déguisements, changements d'identité, sorties et entrées de scène, tout ce vocabulaire auquel nous avons recours laisse clairement apparaître la fibre théâtrale de cette nouvelle. Boiardo recourt par ailleurs massivement aux techniques du théâtre. La fiction d'oralité sur laquelle il a construit son chef-d'œuvre - le fait de s'imaginer comme un conteur face à son public - encourage le recours à ces procédés théâtraux. Si le motif du double, tel qu'il est exploité dans le Miles gloriosus, sert à Boiardo pour imaginer la délivrance de Leodilla des griffes de Folderico, nous savons également qu'il recourt de nouveau à Plaute, notamment à sa comédie des Captivi, pour organiser la reconnaissance de Brandimarte par sa famille, reconnaissance à laquelle est étroitement liée Leodilla. Ces circonstances révèlent d'une part l'extrême soin avec lequel le comte de Scandiano construit ses intrigues; il est en effet impossible de le prendre en défaut : jamais une répétition, jamais la moindre bévue même à plusieurs chapitres de distance. D'un autre côté, la contamination entre nouvelle et théâtre constatée dans le récit de Leodilla range Boiardo parmi les pères fondateurs de la renaissance théâtrale italienne qui, comme chacun le sait, démarre à Ferrare. Avant même l'Arioste et Bibbiena, Boiardo dans son roman a conscience de tout ce que la nouvelle peut apporter au renouvellement et à l'épanouissement de la comédie. Et comme la comédie italienne de la Renaissance se fonde largement sur les nouvelles du Décaméron, on comprend facilement la portée

38. Voir Miles gloriosus, II, 2. Sur l'influence du théâtre de Plaute dans l'Inamoramento, voir M. Villoresi, Da Guarino a Boiardo. La cultura teatrale a Ferrara nel Quattrocento, Rome, Bulzoni, I994. N'oublions pas que Boiardo est également l'auteur d'une pièce, Timone, qui tourne autour du motif de la richesse; voir A. Tissoni Benvenuti et M. P. Mussini Sacchi (éds), M. M. Boiardo, Timone, dans Teatro del Quattrocento, Turin, UTET, I983, p. 47I-555. 
qu’a la décision de Boiardo de recourir à la comédie latine pour dénouer la situation de Leodilla.

À cause précisément de cette contamination entre genre narratif et comédie, la nouvelle de Leodilla se révèle fort intéressante et porteuse d'avenir. Celle de Doristella, peut-être moins novatrice et ambitieuse que la précédente, comporte également un intéressant réemploi d'une nouvelle d'Apulée (Métamorphoses, IX) qui illustre la ruse inventée par Teodoro pour sauver Gambone de la colère d'Osbego voulant le mettre à mort ${ }^{39}$. Cette ruse, inspirée d'un épisode des Métamorphoses, ne concerne plus le couple formé par Doristella et Teodoro, mais permet de souligner la générosité de ce dernier, qui n'abandonne pas à son destin l'humble serviteur par le biais duquel il a pu assouvir son désir. Il s'agit d'une démonstration parmi d'autres de l'humanité des chevaliers de Boiardo, chez lesquels l'ingratitude ne trouve pas grâce.

Les deux nouvelles de Leodilla et Doristella illustrent par conséquent la toute-puissance d'amour que rien ou presque ne peut arrêter. En effet, le dénouement heureux des deux histoires amoureuses tient davantage du hasard que des calculs et de l'habileté des amants. Folderico autant qu'Osbego, une fois qu'ils ont surmonté le dépit de s'être vu soustraire leurs épouses par des stratagèmes bien montés, organisent la rescousse. Tous les deux parviennent à rattraper les amants fugitifs en recourant à la ruse et seul un heureux hasard parvient à libérer définitivement les deux couples d'amoureux des fâcheux jaloux. Folderico, qui venait tout juste de remettre la main sur Leodilla et s'apprêtait à crier victoire, est tué par des brigands, tandis qu'Osbego périt au combat sous les coups de Brandimarte. Dans cet épisode final à rebondissements, le dernier mot est laissé au hasard qui règle le sort des héros du roman chevaleresque autant que celui des humains. Le rôle de la fortune dans ces deux nouvellessœurs tempère ainsi l'idée de toute-puissance d'amour au nom d'un sain réalisme teinté d'optimisme. C'est cet optimisme de fond face à l'adversité que synthétise très bien Doristella, narratrice et personnage de la nouvelle, lorsque, se croyant libre de s'en aller avec Teodoro, elle se voit rattrapée par Osbego : «Ne crois pas que cette nouvelle frayeur qui m'était arrivée ébranlât mon courage; je défiai à nouveau mon sort en me disant que la fortune sourit aux audacieux ${ }^{40}$.»

39. Rappelons-nous que Boiardo avait vulgarisé les Métamorphoses d'Apulée; sur ce volgarizzamento, voir E. Fumagalli, Matteo Maria Boiardo volgarizzatore dell'Asino d'oro. Contributo allo studio della fortuna di Apuleio nell'Umanesimo, Padoue, Antenore, I988; M. Acocella, L'Asino d'oro nel Rinascimento. Dai volgarizzamenti alle raffigurazioni pittoriche, Ravenne, Longo Editore, $200 \mathrm{I}$.

40. II XXVI, 5I, v. I-4 : "Non creder già che per questa paura/ Ch'era incontrata, io me fosse smarita, / Ma più volte me posi ala ventura,/ Dicendo: "A l'animosi il ciel aita!" " 


\section{Les nouvelles de Tisbina et de Narciso}

Les deux nouvelles qu'il nous reste à examiner, celle d'abord d'Iroldo, Tisbina et Prasildo, et ensuite celle de Narciso, ne relèvent pas d'une catégorie identifiable, aussi les examinerons-nous séparément. Dans la première est illustrée une sorte de compétition de courtoisie entre deux chevaliers qui convoitent chacun la même dame, Tisbina ${ }^{41}$. Dans la deuxième, en revanche, sont abordées les questions de la discourtoisie et de l'amour non partagé ou impossible. Comme la nouvelle de Tisbina compte parmi les parties les plus étudiées du poème de Boiardo, bornons-nous à quelques considérations d'ensemble.

Le sujet de cette nouvelle s'inspire de la $\mathrm{X}^{\mathrm{e}}$ journée du Décaméron où il est question de ceux et de celles qui se sont illustrés pour leur courtoisie et magnanimité en amour ou dans d'autres domaines. Mais, d'entrée de jeu, nous constatons que le comte de Scandiano détourne habilement l'esprit de la dixième journée à ses propres fins. En effet, Boiardo situe l'action de sa nouvelle dans la ville de Babylone et appelle son hérö̈ne Tisbina, diminutif de Thisbée, éléments propres à évoquer un lien quelconque avec l'histoire tragique de Pyrame et Thisbée qui se déroule justement à Babylone (Ovide, Métamorphoses, IV, 55-I66). Mais en réalité la nouvelle de Tisbina n'a rien à voir avec l'épisode ovidien, tant pour la lettre que pour l'esprit. L'auteur qui a le plus influencé Boiardo dans la rédaction de cette nouvelle est certainement Boccace, non seulement comme auteur du Décaméron mais aussi comme auteur du Filocolo et des deux poemetti Filostrato et Teseida. Plus précisément, le principal modèle de référence de la nouvelle de Tisbina est la cinquième nouvelle de la $\mathrm{X}^{\mathrm{e}}$ journée du Décaméron, qui raconte l'histoire d'Ansaldo et de Madonna Dianora.

La nouvelle de Boccace non moins que celle de Boiardo concerne un noble chevalier (il est défini "barone» dans les deux cas), amoureux d'une dame déjà liée à quelqu'un d'autre. Dianora, l'héroïne de Boccace, est mariée avec Gilberto, homme riche et respecté ("un gran ricco uomo»), tandis que celle de Boiardo, Tisbina, est liée à Iroldo, lui aussi chevalier. Dianora est convoitée par Ansaldo et Tisbina par Prasildo. Chez Boccace, le mari et l'amant semblent avoir le même statut social, alors que chez Boiardo on a la nette impression que l'amant est socialement plus haut

4I. Sur cette nouvelle, voir au moins la subtile analyse de W. Moretti, «Una novella boccaccesca dell'Orlando innamorato", dans G. Anceschi (dir.), Il Boiardo e la critica contemporanea, Atti del convegno di studi su M. M. Boiardo, Florence, Olschki, 1970. Dorénavant nous indiquerons cette nouvelle sous la forme abrégée de «nouvelle de Tisbina». 
placé que le fiancé, détail qui met ce dernier dans une position d'infériorité $^{42}$. Lautre divergence remarquable concerne la manière dont les deux hommes tombent amoureux. Rien n'est dit à ce sujet dans la nouvelle du Décaméron, alors que Boiardo accorde une grande attention à ce moment, exactement comme le faisait Boccace dans Filostrato, petit poème en ottava rima consacré à l'amour malheureux de Troiolo pour Criseida. Dans Filostrato, le poète s'attarde à décrire le moment où l'amoureux repère la femme choisie au cours d'une cérémonie publique. Dans la nouvelle de l'Inamoramento, Boiardo consacre un bon nombre de vers à la description du jeu de société complexe et érotique pendant lequel Prasildo tombe amoureux de Tisbina ${ }^{43}$. D'un côté, c'est-à-dire dans la Ve nouvelle de la journée $\mathrm{X}$ du Décaméron, le ton est d'emblée digne et grave, conformément à la nature des protagonistes de l'histoire, jusqu'au bout réservés et conscients de leur rang. La nouvelle de Boiardo en revanche débute dans une atmosphère de fête courtoise légère, qui offre les conditions idéales à l'épanouissement de l'amour. Et Boiardo de décrire avec force détails réalistes les sensations de Prasildo qui sent naître en lui l'amour lorsque, jeux oblige, il garde sa tête dans le giron de Tisbina ${ }^{44}$.

Autant Ansaldo que Prasildo se servent d'une messagère pour essayer de plier les dames qu'ils chérissent à leur vouloir, mais là aussi les divergences sont nombreuses. Chez Boccace, la messagère, désignée «buona femina", ne dit rien ou plutôt ne peut rien dire à Dianora, femme noble et vertueuse qui ne tolère pas qu'on lui parle d'une passion malhonnête. Dianora ne répond à la messagère d'Ansaldo que pour se débarrasser d'elle et en même temps de son mandataire. Ce n'est qu'à cette fin qu'elle a l'idée de la mission impossible dont celui-ci doit s'acquitter pour pouvoir bénéficier de son amour. Rien dans le discours net et sans fioritures que Dianora adresse à la messagère ne laisse planer le moindre doute sur la nature de ses intentions $\left(X_{5}, \$ 6\right)$. Cette $V^{e}$ nouvelle, comme d'ailleurs toutes les autres de la journée $\mathrm{X}$, est exempte du moindre sous-entendu misogyne ou grivois. Dans la nouvelle de Boiardo, la situation est tout autre. La messagère de Prasildo invite Tisbina à profiter de l'amour d'un si noble baron et à ne pas laisser passer en vain sa jeunesse sans goûter aux

42. Iroldo est simplement défini «un cavalier» (I XII, 5, v. I), tandis que Prasildo est défini «un Baron [...]/ Di Babilonia stimato il magiore" (I XII, 6, v. I-2).

43. Le jeu prévoit que Prasildo mette sa tête dans le giron de Tisbina. Lorsqu'il est dans cette position, quelqu'un le frappe et il doit deviner qui l’a frappé sans se retourner. Pour la description précise du jeux, voir Inamoramento, note 7 , p. 362 .

44. "Standoli in grembo sente sì gran foco/ Nel cor, che non l'avrebe mai pensato,/ Per non indovinar mette ogni cura, / Ché de levarsi quindi avìa paura. " (I XII, 8, v. 5-8.) 
plaisirs de l'amour (I XII, I4). Des discours similaires sont tenus par le messager de Troiolo à Criseida, la femme que celui-ci convoite. Tisbina, à la différence de celle-ci, ne cède pas aux flatteries de la messagère, mais le fait qu'elle écoute des discours concernant la passion et l'érotisme la rend déjà moins digne, moins intègre et plus potentiellement infidèle que ne l'est Dianora à laquelle ce type de propos ne peut être tenu. Autre divergence de taille entre les deux nouvelles : les conditions dans lesquelles est lancé le défi à l'amoureux. Comme nous l'avons anticipé, dans le récit de Boccace, Dianora, lassée de la cour insistante d'Ansaldo, prie la messagère de rapporter à celui-ci sa requête. Dans la nouvelle de Boiardo, Tisbina et Iroldo surprennent dans un bosquet Prasildo en train de pleurer et de se désespérer pour son amour impossible. Au moment où celui-ci dégaine son épée pour mettre fin à ses jours, Tisbina, d'accord avec Iroldo, sort comme par hasard du fourré et lui propose d'accomplir une mission impossible qui lui permettra, en cas de succès, d'obtenir son amour. La source de cette mise en scène de Boiardo est encore Boccace, plus précisément Teseida, autre poème en ottava rima de Boccace, où Arcita, seul dans un bosquet, se désespère de ne pas être aimé d'Emilia. Un autre écart significatif sépare les deux récits, puisque si Dianora décide seule de la manière dont elle va se débarrasser de la cour d'Ansaldo, chez Boiardo, la décision sur la manière d'éloigner Prasildo est prise de commun accord par les deux amoureux. Cette circonstance finit par faire d'Iroldo le complice de Tisbina et affecte par là en quelque sorte sa dignité d'amoureux en titre. En effet, il écoute sans broncher un autre homme déclarer qu'il est amoureux de sa dame, et au lieu de se manifester pour lui demander raison de ses paroles, il concocte avec son amoureuse une proposition mensongère et tordue. Le mari de Dianora, en revanche, ignore tout de ce marché, qu'il n'aurait peut-être pas approuvé. Sa dignité maritale n'en est donc pas affectée.

Boiardo s'écarte également de Boccace quant à la nature de la mission impossible. Celle-ci n’a rien à voir avec ce que Dianora demande à Ansaldo; la dame lui demande en effet de faire apparaître «en janvier un jardin aussi beau qu'au mois de mai» ("un giardino di gennaio bello come di maggio»). Il faut remarquer que Dianora ne justifie aucunement sa requête qui procède de son seul bon vouloir, ce qui est une manière de mettre à l'épreuve l'amour du chevalier. Tisbina, pour sa part, envoie Prasildo cueillir une branche d'un arbre qui produit des fruits et des feuilles précieuses. Pour le convaincre à partir, elle invoque d'improbables raisons de survie et d'honneur que, par ailleurs, elle se garde bien d'expliciter. L'arbre dont Tisbina exige une branche, le tronc du trésor ("tronco del tesoro»), se trouve dans 
le jardin de Méduse («Orto di Medusa») lui-même situé très loin. La source de l'épreuve que doit surmonter Prasildo pour obtenir l'amour de Tisbina n'est pas tout à fait avérée. Il semblerait qu'elle provienne d'une contamination entre les exploits de Persée (Ovide, Métamorphoses, IV, 7538oI) et ceux d'Hercule dans le jardin des Hespérides ${ }^{45}$. Boiardo donne une interprétation allégorique de cette aventure qui lui offre une fois de plus l'occasion d'aborder sa thématique préférée, c'est-à-dire celle de la relation entre l'amour et la richesse, entre les biens matériels et la générosité et libéralité propres à l'éthique chevaleresque. En effet, on entre dans le jardin de Méduse par la porte de Pauvreté et on en sort par celle de Richesse. Autrement dit, Prasildo entre privé d'amour et il en sort riche d'amour; la branche aux fruits précieux symbolise en effet l'amour qu'a gagné le chevalier qui a su s'acquitter par amour d'une mission qui paraissait impossible. L'autre élément que nous nous bornerons à souligner concerne Méduse; Boiardo suit une interprétation médiévale qui relie Méduse à l'oubli et non à la pétrification de celui qui regarde son horrible visage. Selon nous, dans ce contexte, il faut entendre l'oubli dans un double sens. Pour le chevalier, l'oubli est l'oubli de soi, c'est-à-dire le fait de ne pas accomplir d'exploits exceptionnels. Prasildo a encouru ce risque lorsqu'il se désespérait pour son amour sans agir, mais Tisbina est venue le sortir de sa torpeur en lui proposant cette mission impossible qui est l'énième variation de l' "Omnia vincit amor». Pour Tisbina, en revanche, le motif de l'oubli a une valeur thérapeutique : elle espère simplement qu'en envoyant Prasildo dans une contrée très éloignée, il finira par l'oublier ${ }^{46}$.

Contre toute attente, Ansaldo comme Prasildo réussissent la mission que leur avait confiée la dame aimée. Les réactions des deux héroïnes face à ce succès divergent considérablement d'une nouvelle à l'autre. Dianora révèle à son mari la promesse imprudente faite à Ansaldo. Le mari, considérant la pureté de l'intention de la femme ("considerata la pura intenzion della donna»), lui accorde de respecter la promesse faite à Ansaldo, en faisant preuve d'une grande magnanimité, mais surtout de confiance et de respect à l'égard de sa femme ${ }^{47}$. Les personnages de la nouvelle de Boccace sont calmes et dignes. Après avoir constaté qu'Ansaldo a réussi ce qu'elle

45. Pour les exploits d'Hercule, Boiardo pourrait s'être inspiré des Fatiche di Hercule de Pietro Andrea de' Bassi; voir Inamoramento, note 30, p. 372.

46. "Hor sapiati che Hiroldo e la sua dama/ Mandavano Prasildo a quel giardino/ Che l'Orto di Medusa ancor si chiama, / Aciò che il molto tempo, al longo andare, / Se agia Tisbina delo animo a trare." (I XII, 30, v. 4-7)

47. Parmi les motivations qui poussent le mari de Dianora à lui permettre de respecter la promesse faite à Ansaldo, il y a aussi la peur du negromante auquel Ansaldo s'était adressé pour réaliser ce que la femme avait demandé : Décaméron, $\mathrm{X}, 5, \$ 5$. 
croyait impossible, Dianora éprouve du chagrin; elle ne se plaint ni ne se lamente, mais décide de tout raconter à son mari sans faire de drames: «elle lui révéla tout avec ordre» ("ordinatamente gli aperse ogni cosa»). Toute cette situation délicate est donc abordée avec mesure et sans excès par Dianora et son mari. Le lendemain de l'aveu à Gilberto, Dianora, «sans trop s'orner» («senza troppo ornarsi»), c'est-à-dire très simplement vêtue, détail important, et accompagnée par une femme de chambre et deux personnes de sa famille («due suoi famigliari»), se rend chez Ansaldo pour tenir sa promesse. Celui-ci la reçoit dans une belle chambre éclairée par un grand feu de cheminée et, autre détail important, «sans manifester la moindre concupiscence» ("senza alcun disordinato appetito») à l'égard de Dianora. La noblesse et la dignité dont ont fait preuve Dianora et son mari se communique à Ansaldo.

Il en va autrement chez Boiardo. Ayant appris le succès de Prasildo, Tisbina et Iroldo commencent à se plaindre ensemble, voire à s'accuser réciproquement de la mauvaise idée d'avoir confié à Prasildo une mission dont il a su s'acquitter ${ }^{48}$. Iroldo ne peut pas, bien évidemment, empêcher Tisbina de maintenir sa promesse, car cela serait contraire aux principes de la courtoisie et de la chevalerie, mais il préfère mourir plutôt qu'affronter le tourment de la jalousie. En somme, par sa réaction désespérée, Iroldo d'un côté montre clairement qu'il nourrit des doutes tant à l'égard de la vertu de Tisbina que de celle de Prasildo, de l'autre il fait preuve d'une certaine faiblesse de caractère face à l'épreuve de la jalousie. Or, ce dernier aspect n'est pas du tout effleuré chez Boccace, même si Gilberto aborde ouvertement l'éventualité d'un rapport charnel entre Dianora et Ansaldo ${ }^{49}$. En somme, force est de constater un décalage entre le milieu courtois qui encadre l'histoire de Tisbina et Iroldo et le caractère des héros qui n'est pas à la hauteur de leur rang social ni de leurs idéaux. Encore une fois, le personnage le plus proche d'Iroldo n'est pas Gilberto, mais plutôt Troiolo, le héros plaintif du Filostrato du jeune Boccace, victime d'une passion amoureuse qu'il ne sait ni contrôler ni dominer ${ }^{50}$.

Ne sachant pas comment sortir de l'impasse où les a jetés l'exploit de Prasildo, les deux malheureux amants en viennent à la décision d'absorber un poison. Tisbina se rendra bien chez Prasildo, mais celui-ci ne pourra

48. «Hiroldo sventurato, hor che farai/ Dapoi che avrai la tua Tisbina persa?/ Ben che tu la cagion data te n’hai: / Tu nel mar di sventura m'hai sumersa!» (I XII, 46, v. I-4.)

49. "Voglio che tu a lui vada e [...] dove altramenti non si potesse, per questa volta il corpo ma non l'animo gli concedi." (X, $\left.5, \S_{1} 6\right)$ Nous citons d'après G. Boccaccio, Decameron, édité par V. Branca, Turin, Einaudi, I992, 2 vol.

50. Rappelons-nous que Troiolo, abandonné par la femme aimée qui lui préfère un autre prétendant, finit par mourir des suites du chagrin amoureux. 
jouir d'elle que pendant quelques instants au mieux, car ensuite elle mourra sous l'effet du poison. Autre différence de taille déjà évoquée : si Dianora se rendait en compagnie chez Ansaldo, Tisbina, en revanche, se rend toute seule chez Prasildo. Une fois en présence d'Ansaldo, Dianora, triste et honteuse, lui expliquait qu'elle se rendait chez lui par la volonté de son mari qui avait davantage respecté la passion de l'amant que son propre honneur et celui de sa femme. Tisbina dit aussi à Prasildo qu'elle est venue chez lui par devoir et non par amour et lui avoue ensuite qu'elle a absorbé un poison. Cet aveu désespère Prasildo et l'offense, car cela veut dire que Tisbina n'a confiance ni en sa courtoisie ni en sa magnanimité. Autrement dit, elle n'a pas même été effleurée par l'idée que Prasildo aurait pu renoncer par courtoisie au lourd tribut demandé, se satisfaisant uniquement du plaisir d'avoir rendu un service à la femme aimée. Voici donc que l'indignation de Prasildo face à la méfiance de Tisbina sur la pureté de ses intentions jette une nouvelle ombre sur la vertu des héros. Prasildo, toutefois, se montre bien plus courtois que ne l'imaginaient les deux amoureux - mais pouvait-il ne pas l'être après les soupçons sur sa courtoisie? - en se contentant de demander un simple baiser à Tisbina qu'il renvoie chez Iroldo. Et à ce moment Tisbina, très reconnaissante, lui dit qu'elle aurait pu le chérir si elle avait été libre. Ansaldo lui aussi, impressionné par la grande libéralité de Gilberto, qui allait jusqu'à lui offrir sa femme pour honorer la dette que celle-ci avait contracté avec lui, ne prétendait rien de Dianora, pas même un baiser, et la délivrait entièrement de sa promesse en la laissant libre de rentrer chez elle. De plus, il abandonnait toute velléité érotique, voire sentimentale à l'égard de la dame que, dorénavant, il respecterait et aimerait comme s'il s'agissait de sa propre sœur. Dianora, quant à elle, trop heureuse de ce dénouement inattendu, se garde bien de laisser entendre à Ansaldo qu'elle aurait pu l'aimer, car cela aurait résonné comme une insulte à la magnanimité de celui-ci et, surtout, comme une sorte de revirement de la part de la dame dont l'intégrité n'aurait pas été sans faille. Dianora se borne alors simplement à remercier Ansaldo avec une réserve qui frôle la froideur, lui signifiant en même temps qu'elle n'avait jamais douté de sa courtoisie ni de l'intime noblesse de son âme, qui n'aurait jamais pu tolérer un tel marché. Fasciné par l'extrême courtoisie des deux gentilshommes et de la dame, le sorcier qui avait aidé Ansaldo à réaliser la requête de Dianora décide lui-même de renoncer à son dû. Ce détail est bien la preuve du grand pouvoir de la courtoisie qui élève non seulement l'individu qui la possède mais peut engendrer le bien par son exemple. La nouvelle de Boccace se clôt sur le triomphe de cette vertu tout à la fois éminemment humaine et sociale. 
Chez Boiardo aussi la conclusion est heureuse, mais la leçon à tirer de la nouvelle est très différente. Le poison absorbé par Tisbina et Iroldo se révèle en effet être un simple somnifere. La révélation provient du médecin même qui a vendu le poison à Tisbina. La justification qu'il donne pour expliquer cette substitution providentielle est de l'ordre du poncif misogyne : en gros il faut toujours se méfier des femmes, qui plus est d'une femme qui demande un poison. Ce médecin connaissait évidemment l'histoire des Métamorphoses d'Apulée dont s'est inspiré Boiardo lui-même pour l'épisode du faux poison par lequel il agrémente sa nouvelle avec une dernière péripétie ${ }^{\text {II }}$. Iroldo et Tisbina sont donc sains et saufs, mais au contraire de ce qui se passe chez Boccace, la dame ne reste pas avec son premier amoureux. Iroldo, en effet, impressionné par la grande courtoisie de Prasildo, lui cède Tisbina qui s'accommode très bien de cette situation, car, comme le dit Fiordelisa, la narratrice à laquelle il revient également de tirer la morale de l'histoire, toute femme est volubile et puisque Tisbina est une femme, elle s'adapte bien au changement ${ }^{52}$. Iroldo, quant à lui, quitte Babylone pour partir à la recherche de nouvelles aventures qui lui permettront d'oublier Tisbina.

La nouvelle de Boiardo se clôt donc sur cette morale d'une misogynie tout compte fait plutôt bienveillante à l'égard du sexe féminin, dont on relève les faiblesses sans les fustiger. Si dans la nouvelle de Boccace sont mis en scène des personnages intègres, monolithiques, très rationnels et mesurés, chez Boiardo c'est exactement le contraire qui se passe. Les personnages de sa nouvelle, malgré l'ambiance chevaleresque et courtoise où ils sont plongés, sont au fond très ordinaires. Ils se plaignent, sont inconstants et fragiles et en sont conscients comme nous l'avons constaté. L'amour est certainement une force qui pousse l'homme à se dépasser; il peut améliorer l'être humain comme l'a montré l'exemple de Prasildo, toutefois il ne peut pas changer sa nature qui reste soumise à l'incertitude et à l'instabilité. Si Boccace donne une vision idéalisée de la courtoisie, Boiardo préfère faire preuve de réalisme et montrer toute la complexité et l'ambivalence qui caractérisent jusqu'aux actions et sentiments humains les plus élevés. Nous conclurons en rappelant que l'Arétin a réélaboré la nouvelle de Tisbina dans Lo Ipocrito, l'une de ses comédies les plus intéressantes. Transférés dans ce nouveau contexte comique, les personnages de la

5I. Dans le $\mathrm{X}^{\mathrm{e}}$ livre des Métamorphoses d'Apulée, ouvrage que, rappelons-le, Boiardo avait vulgarisé, il est question d'une méchante belle mère qui essaye d'empoisonner son beau-fils qui a refusé sa cour. Par malheur, le poison est absorbé par le jeune enfant de la dame elle-même. Mais, soupçonnant les mauvaises intentions de la femme, le médecin auquel s'était adressé la dame avait remplacé le poison par un somnifère.

52. La conclusion de la nouvelle de Boiardo s'inspire de la nouvelle de Tito et Gisippo (Décaméron, X, 8). 
nouvelle de Boiardo se renouvellent, notamment le personnage féminin. Dans Lo Ipocrito, ce dernier perd toute connotation de faiblesse et d'indécision pour incarner le type de la forte femme qui indique à son amoureux, à vrai dire plutôt effacé, quelle est la voie à suivre pour s'élever à sa hauteur. Évidemment, il y a dans tout cela une forte dose de parodie et de critique sociale, mais le réemploi de l'Arétin offre une confirmation ultérieure de la richesse et de l'intérêt des thématiques abordées dans cette nouvelle de l'Inamoramento ${ }^{53}$.

La septième et dernière nouvelle de l'Inamoramento que nous allons examiner est une relecture du mythe de Narcisse à la lumière des convictions de Boiardo sur l'amour et sa fonction au sein du consortium humain. Brandimarte et Orlando arrivent à un pont où deux chevaliers sont en train de se battre; l'un d'eux veut empêcher l'autre de traverser le pont ${ }^{54}$. Le lieu de l'action est une prairie avec une fontaine en pierre à côté de laquelle se trouve un tombeau avec une épigraphe qui indique que la tombe est la sépulture de Narciso. Rappelons-nous que cette nouvelle, cas unique, est racontée par le narrateur du roman, intermédiaire entre la narratrice Calidora et le public.

Dans la nouvelle, Narciso est présenté comme un damoiseau («damigello») très beau dont tombe amoureuse la reine d'Orient («la Regina d'Orïente»). Le contexte de la nouvelle est romanesque et médiéval : ni les personnages ni les lieux n'ont de liens directs avec le mythe de Narcisse tel qu'il est raconté par Ovide (Métamorphoses, III, 339-5IO). Boiardo s’inspire en effet du Roman de la Rose, plus précisément de la relecture qu'a fait de ce mythe Guillaume de Lorris, auteur, avec Jean de Meun, d'une partie de cette œuvre capitale de la culture médiévaless. Chez Guillaume de Lorris, le mythe se présente dans un cadre romanesque et médiéval conforme à l'esprit de l'Inamoramento et, détail non négligeable, sous la forme d'un récit exemplaire enchâssé dans une narration plus longue, ce qui devait naturellement séduire Boiardo.

Dans le Roman de la Rose Écho, la nymphe qui dans les Métamorphoses d'Ovide tombe amoureuse de Narciso, devient "une dame de haut rang" et chez Boiardo la plus exotique et noble «Regina d'Orïente», ce qui n’est

53. Au sujet de la réutilisation de la nouvelle de Tisbina par l'Arétin dans Lo Ipocrito, nous nous permettons de renvoyer à notre étude "La mort apparente et ses modèles narratifs dans la comédie italienne du XVI ${ }^{\mathrm{e}}$ siècle ", à paraître en 2010 dans la revue Seizième Siècle (numéro spécial consacré au théâtre de la Renaissance).

54. L'identité des chevaliers est dévoilée à la fin de la nouvelle : il s'agit d'Isolieri, le champion de Calidora et de Sacripante (II XVII, 65, v. 5-8).

55. Guillaume de Lorris et Jean de Meun, Le Roman de la Rose, édité, présenté et annoté par A. Strubel, Paris, Librairie Générale Française, 1992, pour l'histoire de Narcisse, voir v. I437-I507, p. II8-I22. 
pas sans rappeler Angelica, elle aussi princesse asiatique. Autant dans le Roman de la Rose que chez Boiardo, Narciso prend conscience de sa beauté en se regardant dans l'eau de la fontaine, alors que chez Ovide le jeune homme assoiffé se désaltérait dans une source naturelle dont l'eau n'était pas enfermée dans un ouvrage humain. Il s'agit d'un élément hautement significatif. La source indique l'amour et les pouvoirs de l'amour sur les humains; l'enfermement des eaux souligne l'impossibilité de se soustraire à l'amour. Boiardo accentue la nature mortifère de la fontaine en lui associant le tombeau de Narciso, détail absent du Roman de la Rose où une épigraphe gravée sur la fontaine elle-même rappelle les faits. Le comte de Scandiano suit en revanche Guillaume de Lorris lorsqu'il précise qu'avant de mourir de chagrin, la Regina d'Oriente tient des propos de vengeance à l'encontre de Narciso. Cette invocation, adressée à Dieu dans le Roman de la Rose, a un destinataire plus profane chez Boiardo, le dieu d'amour et les cieux.

Une fois épuisée l'histoire de Narciso proprement dite, la nouvelle de Boiardo n'est pas pour autant terminée, car l'auteur lui donne une suite tout à fait personnelle, qui souligne l'actualité du mythe de Narcisse pour le propos qu'il entend développer dans la nouvelle. Le comte de Scandiano imagine en effet qu'une fée, Silvanella, tombe amoureuse de la dépouille du beau Narciso. La série des amours vains inaugurée par Narciso se poursuit ainsi avec Silvanella amoureuse d'un mort, cas emblématique d'amour impossible. Comme Narciso, Silvanella persiste dans sa passion inutile jusqu'à en mourir. Mais avant de s'éteindre, la fée, après avoir enfermé le beau corps tant aimé de Narciso dans un tombeau, ensorcelle la source qui se trouve à côté de la sépulture. Tous ceux qui s'y pencheront verront ainsi surgir des images alléchantes de beauté, de jeunesse et d'amour qui les perdront car ils n’arriveront plus à s'en détacher et périront consommés par l'amour d'une illusion ainsi qu'il advint à Narciso. De ce triste sort est victime Larbino, l'amoureux de Calidora, la narratrice, qui dès lors a décidé de faire garder la source pour éviter que d'autres encourent le même destin tragique que son Larbino bien aimé.

La source de Narciso est en somme un vrai "miroir périlleux" auquel Boiardo n'attribue pas la valeur religieuse que lui donnait l'auteur médiéval. Chez Boiardo, le miroir périlleux indique simplement toute forme d'amour vain contre lequel il veut mettre en garde. Vain est l'amour de Narciso qui, trop épris de lui-même, ignore l'existence même de l'amour. Mais vain est aussi l'amour de la Reine d'Orient, qui se consomme pour qui ne la paie pas de retour, sans parler des cas de Silvanella et de Larbino qui incarnent l'amour pour des objets impossibles ou sans consistance, c'est-à-dire 
l'illusion de l'amour. Boiardo condamne donc toute sorte d'amour qui engendre des souffrances en amoindrissant les capacités humaines, car de son point de vue, l'amour est une force éminemment positive qui épanouit l'homme en le mettant en accord avec la société et la nature. Un amour qui enfermerait le sujet sur lui-même, le coupant de la communauté des humains, qui engendrerait la mort et non la vie et qui s'épuiserait dans les plaintes et l'isolement, ne mérite pas le nom d'amour. Voilà, en substance, la leçon très simple qui émerge de cette nouvelle et qui est annoncée dès le début par l'épigraphe gravée sur la tombe de $\mathrm{Narciso}^{56}$. Boiardo repousse l'amour narcissique, absolument contraire à la philosophie active et énergique qui anime l'Inamoramento.

Rappelons-nous que Boiardo avait donné une tout autre valeur au mythe de Narcisse dans les Amorum Libri Tres, son chansonnier amoureux. Ici, le mythe a une signification plus vaste que dans la nouvelle et il symbolise la vanité de l'amour humain par opposition à l'amour de Dieu. Cette interprétation du mythe est conforme au sens général de la chanson qui le contient. Celle-ci constitue en effet l'avant-dernière composition du chansonnier où il est d'usage que le poète, depuis Pétrarque, prenne définitivement congé des vaines amours humaines pour se tourner vers des réalités d'ordre spirituel et divin ${ }^{57}$. Dans la nouvelle, les effets pervers de l'amour vain sont interrompus par la décision généreuse de Calidora de faire garder la source, afin qu'elle ne fasse pas d'autres victimes. Victime elle-même du pouvoir maléfique du miroir périlleux, Calidora, plus magnanime que la reine d'Orient et Silvanella, est la première à ne pas se venger de son malheur sur les autres; au contraire, elle veut que son malheur serve de leçon afin d'interrompre le pouvoir maléfique de la source. La décision généreuse et désintéressée de Calidora enrichit la leçon de la nouvelle d'une dimension de piété et d'altruisme provenant du christianisme que Boiardo n'affiche pas mais qui sous-tend tout son roman. Les valeurs de la chevalerie et de la courtoisie, avec leurs idéaux de générosité, d'aide aux plus faibles, de désintéressement et de respect humain, ont leurs racines dans un humanisme chrétien qui pour Boiardo est escompté. Cette nouvelle, pratiquement la dernière de l'Inamoramento puisque ne lui succède que celle de Doristella - réplique de celle de Leodilla qui n'ajoute donc rien de nouveau -, est en quelque sorte le bilan de ce qui

56. "[...] Ben è quel'alma vana/ Qual s'invagisse mai dil suo bel viso!/ Quivi è sepolto il giovane Narcyso " (II XVII, 49, v. 6-8); ces vers sont gravés sur le tombeau.

57. Voir M. M. Boiardo, Amorum Libri Tres, édité et annoté par C. Micocci, Milan, Garzanti, 2006 (I ${ }^{\text {re }}$ éd. I990), chanson $n^{\circ} 179$, v. 49-60. 
a été dit sur l'amour dans les six précédentes. Que Boiardo y attache une importance particulière est confirmé par le soin formel qui la caractérise. Ce soin émerge notamment à la fin de certaines strophes où sont énoncées des vérités générales sur l'amour que l'auteur souligne en recourant à des tournures stylistiques et rhétoriques recherchées. Le geste altruiste de Calidora, qui annule l'effet du miroir périlleux, rappelle ainsi la nature fondamentalement altruiste, désintéressée et bénéfique de l'amour, lequel ne porte des fruits que lorsqu'il est mis au service d'autrui. Et ce dernier aspect est essentiel pour le comte de Scandiano, qui attribue à l'amour une haute mission civilisatrice et culturelle, car, comme l'a bien formulé Claudia Micocci, «si la parole est à l'origine de la civilisation humaine, c'est Amour qui la pousse à se manifester ${ }^{58} »$.

Nous avons pu constater que les nouvelles de l'Inamoramento ne sont pas des digressions sans liens avec l'action principale, même si certaines comportent vis-à-vis de celle-ci un plus haut degré d'autonomie que d'autres. Reliées à l'action majeure par des liens structurels, les nouvelles le sont également au niveau sémantique, car comme il était prévisible, elles reprennent et approfondissent des thématiques traitées aussi dans le roman. En effet, le simple fait d'isoler ces thématiques à l'intérieur d'une narration spécifique donne à ces motifs un relief qu'ils n'auraient pas ou beaucoup moins s'ils n'avaient pas été éclairés à la lumière des nouvelles. L'hypothèse selon laquelle le retranchement des nouvelles du roman de Boiardo serait sans conséquence ne nous semble donc pas pertinente et nous espérons l'avoir montré en soulignant les rapports complexes et subtils qui existent entre la narration principale et les digressions. D'un côté les nouvelles sont soudées au roman qui les contient et de l'autre elles sont autonomes en raison de leur haut degré d'achèvement - pour la forme comme pour le fond. Cette circonstance est d'autant plus remarquable que l'Inamoramento est une œuvre ouverte, et cela par-delà son état d'inachèvement. Nous avons en effet pu observer que la manière dont Boiardo se sert de la nouvelle contribue à faire évoluer le roman vers une forme plus moderne et accomplie. Le défi relevé par le comte de Scandiano d'agrémenter avec des nouvelles l'intrigue du roman chevaleresque comportait, il est vrai, un certain nombre de difficultés. Tout d'abord, il fallait adapter la nouvelle, caractérisée depuis Boccace par un certain réalisme du cadre, des personnages et des situations, au contexte merveilleux du roman chevaleresque 
arthurien. Ensuite, le roman lui-même était un genre populaire, aimé par le public, y compris par un public raffiné comme celui auquel s'adressait Boiardo, mais qui suscitait la méfiance des doctes. Faut-il encore rappeler l'anathème lancé par Pétrarque contre "quei che le carte empion di sogni» (Triumphus Cupidinis, III, v. 79)? N'oublions pas non plus qu’à l'époque où Boiardo composait l'Inamoramento il n'existait pas de cadre normatif ni théorique pour orienter un auteur dans son cheminement vers l'accomplissement formel. Autrement dit, Pietro Bembo n'était pas encore là pour indiquer quels modèles imiter et quelles techniques suivre en vue de produire une œuvre littéraire accomplie. Et les débats sur l'unité d'action, sur la place des digressions et du merveilleux dans le poème héroïque et dans le roman, qui devaient contribuer à la naissance du roman moderne, étaient encore bien loin ${ }^{59}$. Ces rappels suffisent à souligner la charge novatrice de l'initiative de Boiardo. Car en choisissant d'insérer des nouvelles dans le moule du roman, le comte de Scandiano a l'intuition géniale grâce à laquelle le roman chevaleresque évolue de la structure paratactique - impliquant l'avancement de l'action par ajouts successifs - à une structure qui pose le principe d'une hiérarchie entre les différentes parties composant le roman. Boiardo est ainsi l'un des premiers à comprendre que la force du roman moderne repose sur sa capacité non pas à tolérer des ajouts, comme dans le roman chevaleresque traditionnel, mais à intégrer des éléments narratifs divers à l'intérieur de son propre corps. Exemplaire est à cet égard sa capacité - à l'œuvre aussi dans certaines de ses nouvelles, nous avons pu le constater - à contaminer et à faire cohabiter des genres différents tels que le roman, la nouvelle et le théâtre. Dans l'Inamoramento, la nouvelle fonctionne comme un nœud, un point de fixation dans l'élaboration du fil narratif. Elle est en somme le moyen dont dispose l'auteur pour revenir sur certains épisodes, personnages et motifs, et leur donner un relief particulier dans l'action. Sans les nouvelles, le roman de Boiardo ressemblerait davantage à une succession d'épisodes, et il n'aurait pas le relief et la variété qui contribuent à lui donner sa force et à faire son originalité.

59. Sur les débats de la Renaissance autour des genres narratifs, voir M. Stanesco, «Premières théories du roman. Les folles amours des paladins errants», Poétique, nº 70, 1987, p. I67-I80; N. Ordine (éd.), Traités sur la nouvelle à la Renaissance. Bonciani, Bargagli, Sansovino, Turin-Paris, Aragno-Vrin, 2002; Les poétiques italiennes du "roman». Simon Fornari, Jean-Baptiste Giraldi-Cinzio, Jean-Baptiste Pigna, traduction, introduction et notes par G. Giorgi, Paris, Champion, 2005; E. Malato, «La nascita della novella: un'alternativa letteraria borghese alla letteratura cortese", dans La Novella italiana, op. cit., p. 3-45; G. B. Pigna, I Romanzi, édition critique par S. Ritrovato, Bologne, Commissione per i testi di lingua, 1977. 